\title{
Transducers as Gates to the Universe
}

\author{
Ciulin Adam Dan, E-I-A Lausanne, Switzerland
}

\begin{abstract}
The 'main structure and performances' of any physical system may be (nearly) maintained for a given time interval only if this system is kept inside a kind of 'shell' that protects it and only if the necessary (desired) exchange of energy-mass with its environment is realized through some transducers. The subsystem constituted by the shell and transducers constitutes a kind of 'barrier' between this physical system and its environment. Then, humans can exchange energy, mass, and information with their environment only though their available transducers. The paper presents sections about Plank energy, about inertia in rotation, translation, about waves and space dimensions, all based on classical physics and mathematics. These sections present in a different manner some very well-known physical phenomena. Associated with a new and convenient technology, all these models may be considered as innovation strategy importance. Applications may be found in interplanetary telecommunications and treks but also for everyday Earth vehicles and life.
\end{abstract}

\section{KEYWORDS}

Antigravity, Electromagnetic Interaction, Fields, Gravity, Inertial, Reliability of Information, Strategic Information, Time

\section{INTRODUCTION}

In nature, any item interacts with its environment. Due to these interactions that may transfer energy and/or even mass, the structure and also the properties of each items change more or less quickly in time. This process may be considered as being an 'aging process.' For this reason, any actual present configuration of the universe may be considered as being 'ephemerae.' In biology, all items are protected with some kind of 'shell' that is a 'membrane' for bacteria or a 'skin' for mammalians. This 'shell' may protect a living organism only for certain kinds of interactions with their environment but it implies the presence of 'transducers' to realize the necessary desired interactions with this environment. An engine powered by chemical energy needs fuel and an oxidizer to work. Mammalians need nourishment and oxygen to survive. The 'digestive system transducer' ensures the transfer of necessary nourishment and the 'respiration system transducer' ensures the transfer of necessary oxygen from the environment. The respiration rhythm is higher than the nourishment rhythm. Humans has eyes to sense the environmental light, ears to sense the environmental acoustic sounds etc. During their evolution, humans had created in their minds a kind of 'virtual environmental image' that helps them survive. Generally, transducers constitute some 'access gates' between any living organism and its environment. The 'digestive system' and the 'respiration system' which are essential for any living organism to survive, implies for example, that a human must not eat, drink and/or breathe in, some potentially dangerous item. The sex system also constitutes a kind of 'access gate' between living 
organisms and their environment. During the lifetime, our transducers lose their ability and then, at the end of life, we become more or less 'disabled.'

Let now consider a mathematical function $y=F[x]$ associated to a transducer where $x$ may be considered as the input signal, $y$ the output signal and $F[$.$] the transducer function. To 'well$ 'decode the right signal $x$ ' of such a function, the 'inverse function' of this system must also be known. Generally:

$$
F^{(-1)}[F[x]]=x+\varepsilon[x]
$$

and then, if $\varepsilon[x]$ is not smaller that the accepted error, the input signal cannot be 'well decoded'. By analogy with a Phase Lock Loop that may realize such 'inversions', an equivalent loop is (probably) realized by the brain and then, inside the brain, a kind of 'virtual mirror' of ' $x$ ' is built. In this manner, we may probably 'be aware and understand more or less' our environment. This kind of 'virtual environment' is adjusted every day and during sleep, the human brain 'codes' the daily-received information in order to diminish its 'information volume'. Living organisms work using a 'quaternary base' in DNA (Deoxyribonucleic acid) and the 4D space is very special because only polynomials of degrees less than 5 may be solved by algebraic methods. It can also be observed that some special transducers exist only to the mammalians that need them every day to survive. Sometime, a given human may has also some transducers that are 'not well installed' or have some flaw and then cannot be correctly used. Actual technology may help a given human to improve some flaws of his transducers. For example, the British-Catalan artist Neil Harbisson (Wikipedia, Neil Harbisson, 2016) who was 'color blind born' and seen all images only in gray, had accepted in 2004 to graft on his head a cybernetic antenna who let him 'hear the colors'. This antenna is a color transducer connected to a microprocessor that changes input colors into sounds. These sounds are then applied to his skull by means of a special loudspeaker fixed onto the bones of skull. Memorizing the sound equivalent to each color and using his antenna, he is now able to associate colors to each image that he views. Figure 1 shows Neil Harbisson with his cybernetic antenna graft on the skull and a portrait colored by him using Photoshop software.

Based on these ideas, some problems will be analyzed and developed to make a kind of 'bridge' to classical theory in order to realize a better understanding of some physical phenomena.

\section{ABOUT PLANCK ENERGY}

Let us now consider electrical signals modeled mathematically by analytic complex-exponential functions. The analytic signal corresponding to a real given voltage electrical signal $U(t)$ is given by:

$$
z(t)=U(t)+j \cdot \mathrm{H}[U(t)] ; \mathrm{H}[U(t)]=\frac{P V}{\grave{A}} \cdot \int_{-\infty}^{\infty} \frac{U(\tau)}{t-\tau} \cdot d \tau ; j=\sqrt[2]{-1}
$$

where $\mathrm{H}[U(t)]$ is the Hilbert transform of the signal $U(t)$ and ' $P V$ ' means 'principal value of theintegral.' For $U(t) \equiv \operatorname{Cos}(t)$, the corresponding analytic signal is $z(U(t))=\operatorname{Cos}(t)+j$. $\operatorname{Sin}(t)=e^{j . t}$.

One may observe that $\mathrm{H}[z(t)]=-j \cdot e^{j . t}$ and this correspond to the rotation with $\frac{\pi}{2}$ of the signal axis. The Fourier transform that may be considered as a (mean-value) 'time to frequency and/or vice-versa converter' between a time signal $s(t)$ over a given time interval and its frequency spectrum $S(j . \omega)$ is represented by: 
Figure 1. A portrait colored by Neil Harbisson

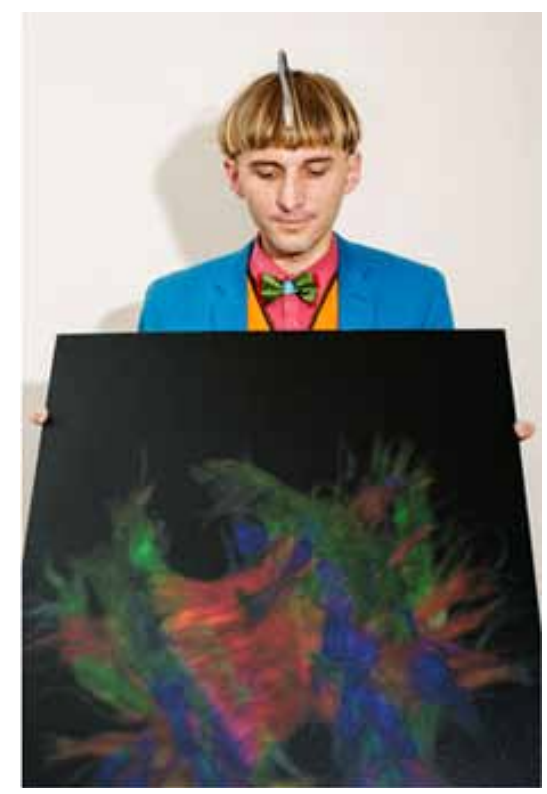

$\left\{\begin{array}{l}S(j \cdot \omega)=\int_{-\infty}^{\infty} s(t) \cdot e^{-j \cdot \omega \cdot t} \cdot d t \\ s(t)=\frac{1}{2 \cdot \pi} \cdot \int_{-\infty}^{\infty} S(j \cdot \omega) \cdot e^{j \cdot \omega \cdot t} \cdot d t\end{array}\right.$

where:

$$
\left\{\begin{array}{c}
\omega=2 . \pi \cdot f \\
f=\frac{1}{T}
\end{array}\right.
$$

and $f, T$ are (respectively) the probabilities of a given frequency and period of the signal $s(t)$. Then, the signal spectrum $S(j . \omega)$ may be considered as being a 'density probability distribution'. The power of signal $U(t)$ is given by:

$$
P(U)=\operatorname{Lim}_{T \rightarrow \infty}\left[\frac{1}{2 . T} \cdot \int_{-T}^{T} U(\tau) \cdot U(t+\tau) \cdot d \tau\right]
$$

The Fourier transformation of $P(U)$ corresponds to its spectral power density:

$$
S_{P(U)}(j \cdot \omega)=\int_{-\infty}^{\infty} P(U(t)) \cdot e^{-j \cdot \omega \cdot t} \cdot d t=S_{U(t)}(j \cdot \omega) \cdot S_{U(t)}^{*}(j \cdot \omega)=\left|S_{U(t)}(j \cdot \omega)\right|^{2}
$$


where by '*' we have denoted the 'complex-conjugate'. Using Parceval theorem, the energy $E_{s}$ of the signal $U(t)$ over the time interval $-\infty, \infty$ results as:

$$
E_{s}=\int_{-\infty}^{\infty}|U(t)|^{2} \cdot d t=\frac{1}{2 \cdot \pi} \cdot \int_{-\infty}^{\infty}\left|S_{U(t)}(j \cdot \omega)\right|^{2} \cdot d \omega
$$

where $|U(t)|^{2}$ may be considered as 'instantaneous energy of the signal $U(t)$ '. It may be observed that the energy $E_{s}$ of a signal on a given (constant) load $\mathrm{Z}$ is given by:

$$
E=\frac{E_{s}}{Z}=\frac{1}{Z} \cdot \int_{-\infty}^{\infty}|U(t)|^{2} \cdot d t
$$

Let us now consider an ideal voltage to frequency converter that have at its input, the signal $|U(t)|^{2}$. At its output, it will result a signal of constant amplitude $\operatorname{Cos}(\phi(t))$ of an instantaneous frequency $f_{U}(t)$ proportional to the amplitude of the input signal $|U(t)|^{2}$. Then ideally, for each instant in time, we may consider that

$$
|U(t)|^{2} \Rightarrow k \cdot f_{U}(t)
$$

where the converter proportionality factor is $k$. Remembering that the function $\phi(t)$ may be considered as being the 'instantaneous phase' results:

$$
\frac{d \phi_{U}(t)}{d t}=\omega_{U}(t)=2 \cdot \pi \cdot f_{U}(t)
$$

where $\omega_{U}(t)$ is its 'instantaneous pulsation'. It may be observed that the output voltage of such a phase/frequency converter is equivalent to a phase/frequency modulator with a 'zero frequency carrier' and may practically have a bloc diagram as in Figure 2. The modified PLL (Phase Lock Loop) from figure 2 uses a symmetrical frequency-to-voltage converter (SFVC), a voltage-to-frequency converter (SVFC), and an (ideal) low-pass filter (LPF) having a frequency bandwidth equal to the input-applied signal to reconstruct $\operatorname{In}(\mathrm{t})$. Ideally, $\mathrm{r}(\mathrm{t})$ will be nearly equal to $\operatorname{In}(\mathrm{t})$ and then, the signal Out(t) at the output of the SFVC bloc will be nearly similar to $\operatorname{Cos}(\phi(t))$. From here, it results that the signal $U_{c}(t)$ at the output of an ideal voltage to phase/frequency converter having as input the signal $|U(t)|^{2}$ will be:

$$
U_{c}(t)=\operatorname{Cos}\left(\phi_{U}(t)\right)=\operatorname{Cos}\left(\int 2 \cdot \pi \cdot k \cdot f_{U}(t) \cdot d t\right)=\operatorname{Cos}\left(2 . \pi \cdot \int|U(t)|^{2} \cdot d t\right)
$$

The mean value of the energy of the signal $U(t)$ over the time interval $T=T_{2}-T_{1}$ corresponds to: 
$\tilde{E}_{T}(U(t))=\frac{1}{T} \cdot \int_{T_{1}}^{T_{2}}|U(t)|^{2} \cdot d t \Rightarrow \frac{k}{T} \cdot \int_{T_{1}}^{T_{2}} 2 . \pi \cdot f_{U}(\tau) \cdot d t=K \cdot \tilde{\phi}_{U}=2 . \pi \cdot K \cdot \tilde{f}_{U}$

where $K$ is a coefficient and $\tilde{\phi}_{U}, \tilde{f}_{U}$ are, respectively, the mean values of the phase and frequency over a time interval $T$. Then, the output signal of such a voltage to frequency converter will be:

$$
\tilde{E}_{t, t+T}(U(t))=2 \cdot \pi \cdot K \cdot \tilde{f}_{U}=K_{1} \cdot \tilde{f}_{U}
$$

which is similar to the Planck-Einstein relation for energy. We remember that Plank-Einstein relation is:

$E_{P}=h . f ; h=6.626070040(81) \cdot 10^{-34} \mathrm{~J} \cdot \mathrm{s} ;$

Relation (13) makes a bridge between the ordinary definition of energy as being a rectangular and/or square product of amplitude, and its new definition which is connected with the mean value of a given instantaneous frequency that may exist in another dimension, the dimension of the output space of an ideal voltage to frequency converter.

A more advanced analysis leads to the observation that any practical signal may be considered as being a real signal that has a limited frequency bandwidth $f_{\max }$ and may also be represented by the sampling theorem. The sampling function $F_{f_{\max }}(t, n)$ is given by:

$$
F_{f_{\max }}(t, n)=\frac{\operatorname{Sin}\left[2 \cdot \pi \cdot f_{\max } \cdot\left(t-\frac{n}{2 \cdot f_{\max }}\right)\right]}{2 \cdot \pi \cdot f_{\max } \cdot\left(t-\frac{n}{2 \cdot f_{\max }}\right)} ; n=1,2, \ldots ;
$$

Figure 2. Bloc diagram of a voltage to frequency converter

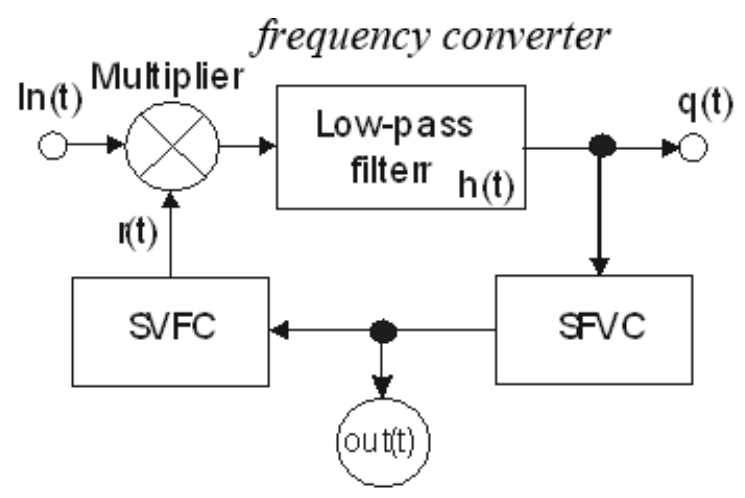


where $t$ is the time. Over its time support, any signal $s(t)$ with limited frequency bandwidth to $f_{\max }$ and limited time support may be represented by:

$$
s(t)=\int_{-R}^{t} s(\tau) \cdot \delta_{T_{m}}(\tau) \cdot h(t-\tau) \cdot d \tau=\sum_{n=1}^{N} s\left(\frac{n}{2 \cdot f_{\max }}\right) \cdot \frac{\operatorname{Sin}\left[2 \cdot \pi \cdot f_{\max } \cdot\left(t-\frac{n}{2 \cdot f_{\max }}\right)\right]}{2 \cdot \pi \cdot f_{\max } \cdot\left(t-\frac{n}{2 \cdot f_{\max }}\right)} ; n=1,2,3, \ldots
$$

where $R$ is a enough higher limit of time in the past, $\delta_{T_{m}}(\tau)$ is the periodic Delta Dirac function, and $h(t)$ is an ideal low-pass filter having the limit frequency equal to $f_{\max }$. From relation (16) it seems that any practical signal source sends into a given physical channel, at any discreet time, $t=\frac{n}{2 . f_{\max }} ; n=1,2,3, \ldots$, discreet amplitude-modulated energy balls, mathematically equivalent to $s\left(\frac{n}{2 . f_{\max }}\right) \cdot \delta\left(\frac{n}{2 . f_{\max }}\right)$. The channel transforms the energy balls into a 'low-pass filtered wave' that travels through. For example, a light source may send 'photons' in a given direction of the 'free space' and it result an equivalent wave that travels into this space. In some special situations (such as with a light sensor) this equivalent wave seems to be transformed back into photons according to so-called 'Einstein photoelectric effect' and is then able to ejects electrons from matter. Of course, this effect results only if the energy of the wave is greater or at least equal to 'work function' (the binding energy of electron to the matter). Then, the sampling function $F_{f_{\max }}(t, n)$ may be considered as a 'base function' that can represent any physical signal. The trigonometric function $\operatorname{Cos}($.$) introduce branches:$

$$
\operatorname{Arg} \operatorname{Cos}[\operatorname{Cos}(t)]=t_{p} \pm 2 . n . \pi ; n=1,2,3, \ldots ;
$$

where $t_{p}$ is the equivalent time argument limited to a single period of the function $\operatorname{Cos}($.$) . Then,$ we may introduce an 'equivalent argument function' by:

$$
G(t)=k_{1} \cdot t-\operatorname{Arg} \operatorname{Cos}\left[\operatorname{Cos}\left[k_{1} \cdot\left(t-\frac{1}{2 \cdot f_{\max }}\right)\right]\right]
$$

where $k_{1}$ is a coefficient. This function is represented in Figure 3.

By introducing the 'switching function $\operatorname{Sign}[\operatorname{Sin}()$.$] ', relation (18) becomes:$

$$
G n(t)=k_{1} \cdot t-\operatorname{Sign}\left[\operatorname{Sin}\left[k_{1} \cdot\left(t-\frac{1}{2 \cdot f_{\max }}\right)\right]\right] \cdot \operatorname{Arg} \operatorname{Cos}\left[\operatorname{Cos}\left[k_{1} \cdot\left(t-\frac{1}{2 \cdot f_{\max }}\right)\right]\right]
$$

which is represented in figure 4. It may be easily observed that the switching function $\operatorname{Sign}[\operatorname{Sin}()$. modify the 'slope' of the function $G(t)$ for some convenient time intervals and then, the time derivative of the function $G n(t)$ corresponds to the periodical Dirac Delta function. From here, relation (16) becomes: 
Figure 3. $G(t)$ function.

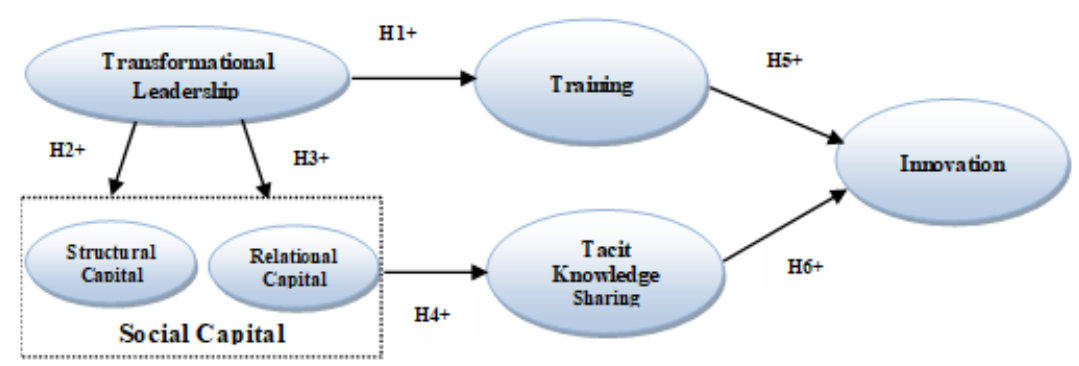

Figure 4. $G n(t)$ ladder function.

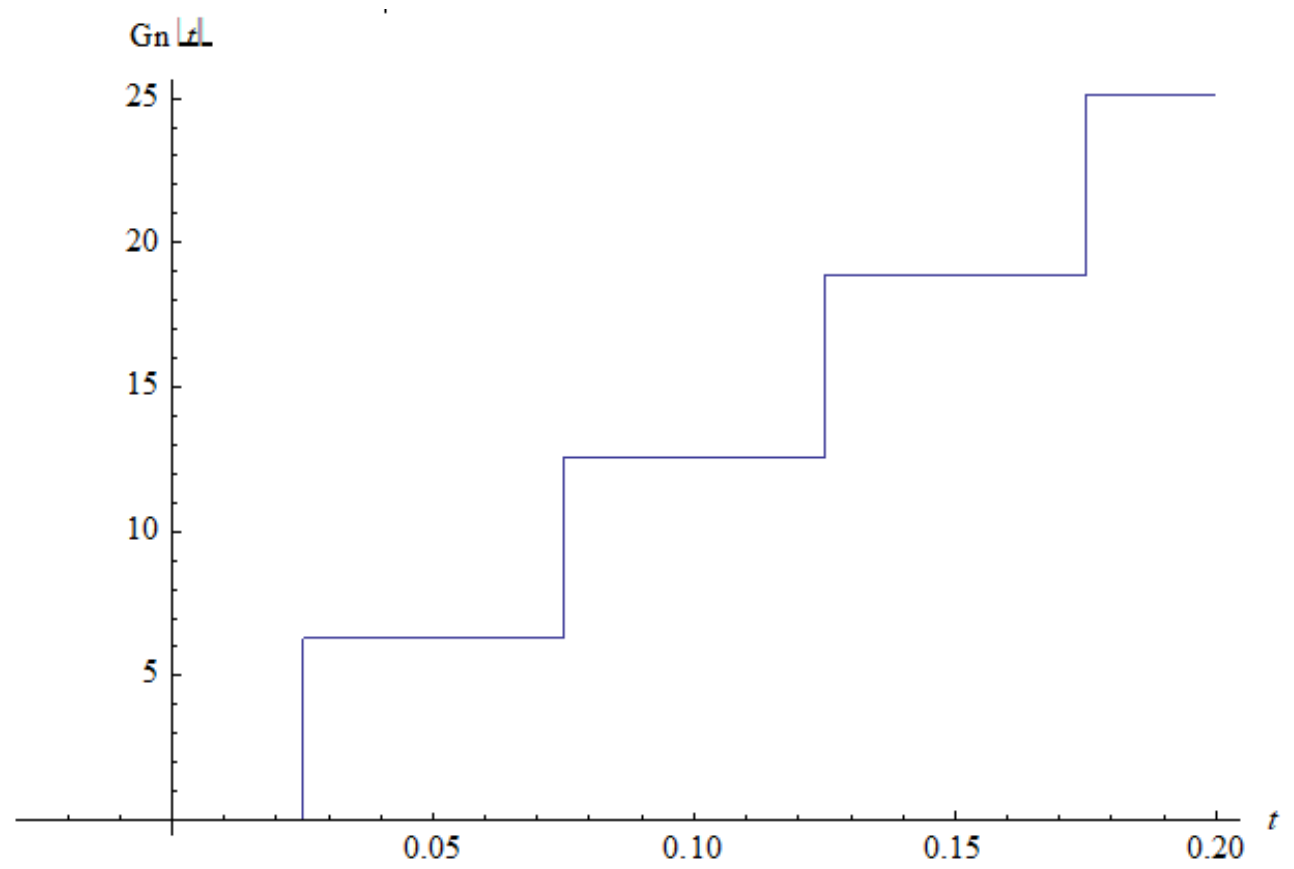

$s(t)=\int_{-R}^{t} s(\tau) \cdot \frac{d[G n(\tau)]}{d \tau} \cdot h(t-\tau) \cdot d \tau$

where the time $t$ is connected with the signal $s(t)$ at the output of the physical channel and the time $\tau$ with the signal inside the filter $h(t, \tau)$ function.

A 'reconstruction sampling function' may be represented by: 
$F f(t)=\frac{\operatorname{Sin}\left(2 \cdot \pi \cdot f_{\max } \cdot t\right)}{2 . \pi \cdot f_{\max } \cdot t}$

and its Fourier spectrum by:

$\operatorname{SFf}(\omega)=\sqrt[2]{\frac{\pi}{8}} \cdot \frac{\operatorname{Sign}[20 \cdot \pi-\omega)+\operatorname{Sign}[20 \cdot \pi+\omega)}{20 . \pi} ;$

Figure 5 shows this 'reconstruction sampling function,' and figure 6 shows its (ideal) Fourier spectrum for $f_{\max }=10$. If (ideally) the frequency bandwidth of the input signal $s(t)$ is exactly equal to the frequency bandwidth of the sampling function $F f(t)$, then the product $s(t) \cdot \frac{d[G n(t)]}{d \tau}$ from equation (20) may be considered as being inside the frequency bandwidth of the signal $s(t)$ because each of its 'amplitude modulated sample with a given constant' has this frequency bandwidth. In fact:

$r(t)=s(t) \cdot F f(t) \Rightarrow R(j \cdot \omega)=\int_{-\infty}^{\infty} s(t) \cdot F f(t) \cdot e^{j \cdot \omega \cdot t} \cdot d t=\frac{1}{2 \cdot \pi} \cdot \int_{-\infty}^{\infty} S(j \cdot \Omega) \cdot S F f(j \cdot(\omega-\Omega)) \cdot d \Omega$

and the time support of the sampling function is 'cut so to be equal with the time support of the input signal.' Then, for a signal time support of 10 seconds:

$$
\begin{aligned}
& \sum_{n} s(t) \cdot F f(t, n)=s(t) \cdot \sum_{n} F f(t, n)=s(t) \cdot 1[t]= \\
& s(t) \cdot\left(\frac{\text { Sin Integral }\left[2 \cdot \pi \cdot f_{\max } \cdot(t+5)\right.}{2 \cdot \pi \cdot f_{\max }}-\frac{\text { Sin Integral }\left[2 \cdot \pi \cdot f_{\max } \cdot(t-5)\right.}{2 \cdot \pi \cdot f_{\max }}\right)=s(t) \cdot 1(t)
\end{aligned}
$$

where the function $1(t)$ is represented in figure 7 . For simulation, the considered input signal $s(t)$ is given by:

$$
\begin{aligned}
& s i(t)=4 . \operatorname{Sin}(2 . \pi . t)+3 \cdot \operatorname{Cos}(2 . \pi \cdot 2.3 . t)+3.5 \cdot \operatorname{Cos}(2 . \pi \cdot 4 \cdot 1 . t)+2.2 \cdot \operatorname{Cos}(2 . \pi .5 .15 . t)+ \\
& \text { 1.5. } \operatorname{Sin}(2 . \pi \cdot 6.3 . t)+1.2 . \operatorname{Sin}(2 . \pi \cdot 8 \cdot 2 . t)+\operatorname{Sin}(2 . \pi .9 . t)
\end{aligned}
$$

Figure 8 show a superposition of the function $1(t)$ in red and $s(t) .1(t)$ in blue. Relations (15) to (21) presents the interpolation of a limited frequency bandwidth signal in a given time interval. The errors of interpolation are shown in figure 9 . Inside the time interval $(0,5)$, these errors are shown in figure 10 . The errors of interpolation over the time interval $(0,10)$ are shown in figure 11. Similar results are obtained in MATLAB, as can be seen in figures 12 through 14. Figure 12 show the sampling function and its spectrum. The instantaneous energy and its 'cumulative sum' over 1 second for a sample function are shown in figure 13. To obtain 'power amplitude samples', we multiply each amplitudes sample of a given input signal by itself. Then, their equivalent mean value frequencies will change in function of this signal. The instantaneous frequency delivered by an ideal amplitude to frequency converter at whom is applied an input corresponding to the instantaneous energy of a given signal will depends also on at the parameters of the used convertor. Figure 14 shows the input 
Figure 5. $F f(t)$ sampling function.

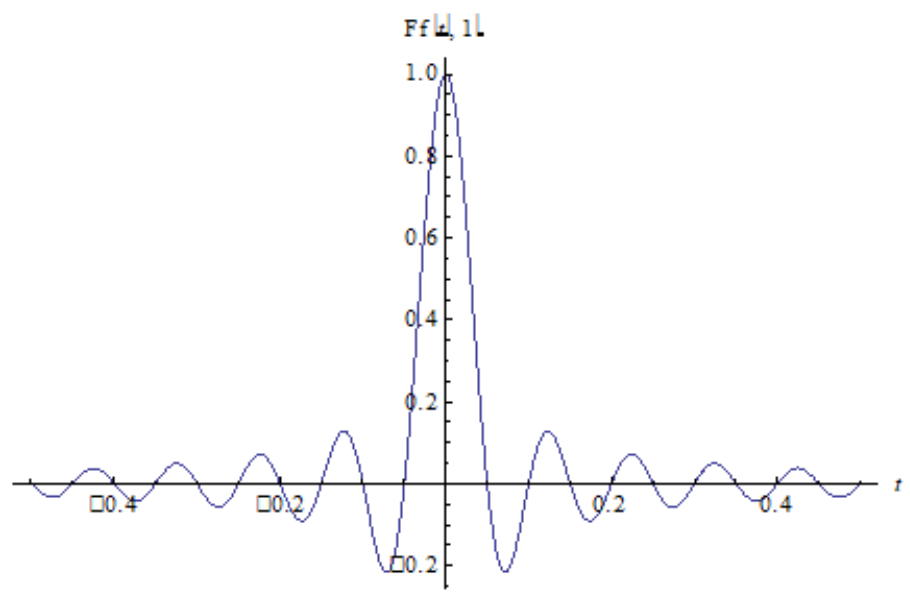

Figure 6. Ideal absolute value spectrum of $F f(t)$

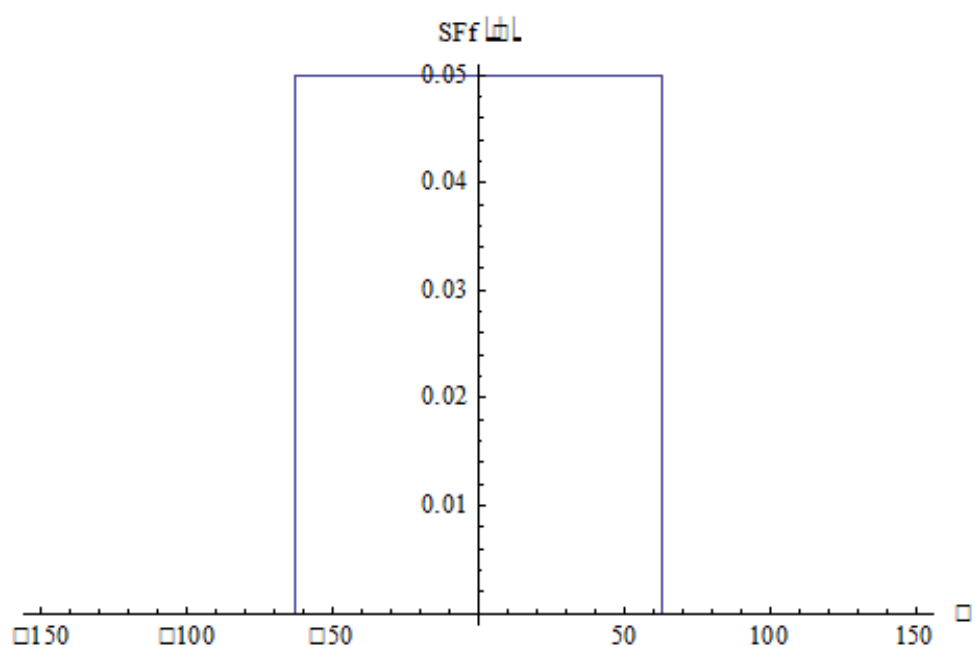

signal, its instantaneous energy and its instantaneous equivalent mean value frequency for an amplitude to frequency converter with $K=2$. From here, the mean values of frequencies for a single sampling function over 1 second and $K=2$ are equal to:

$$
\tilde{f}_{\text {Sam }}=\left.\frac{\tilde{E}_{t, t+1}(F f(t))}{2 . \pi \cdot K \cdot T}\right|_{K=2}=3.9386 \mathrm{~Hz}
$$


Figure 7. 1(t) function

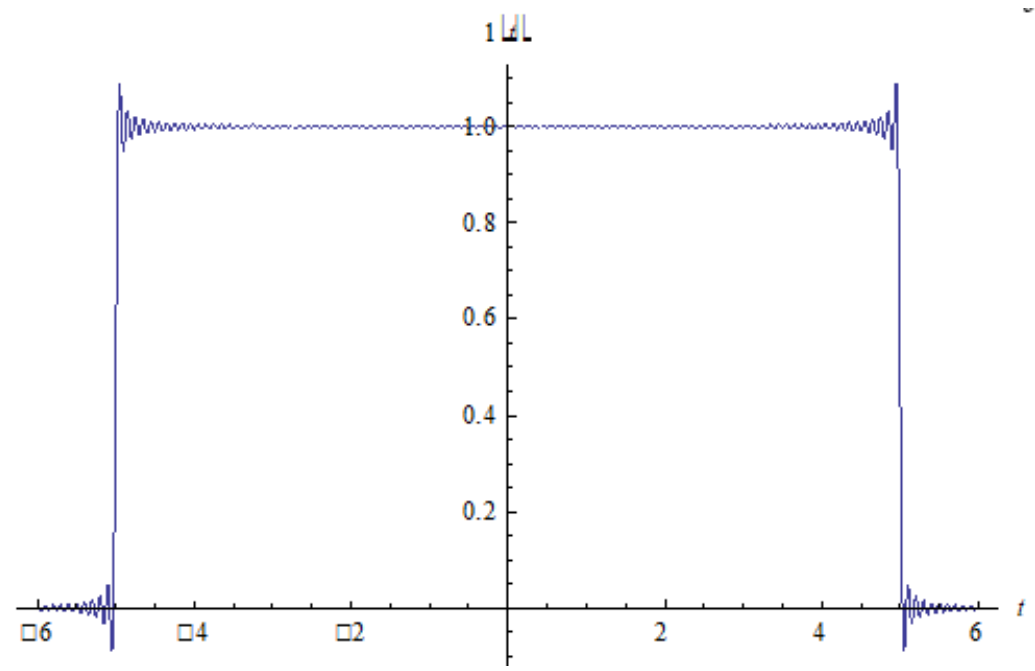

Figure 8. Product of input signal with ' $1(t)$ ' function and the function $1(t)$.

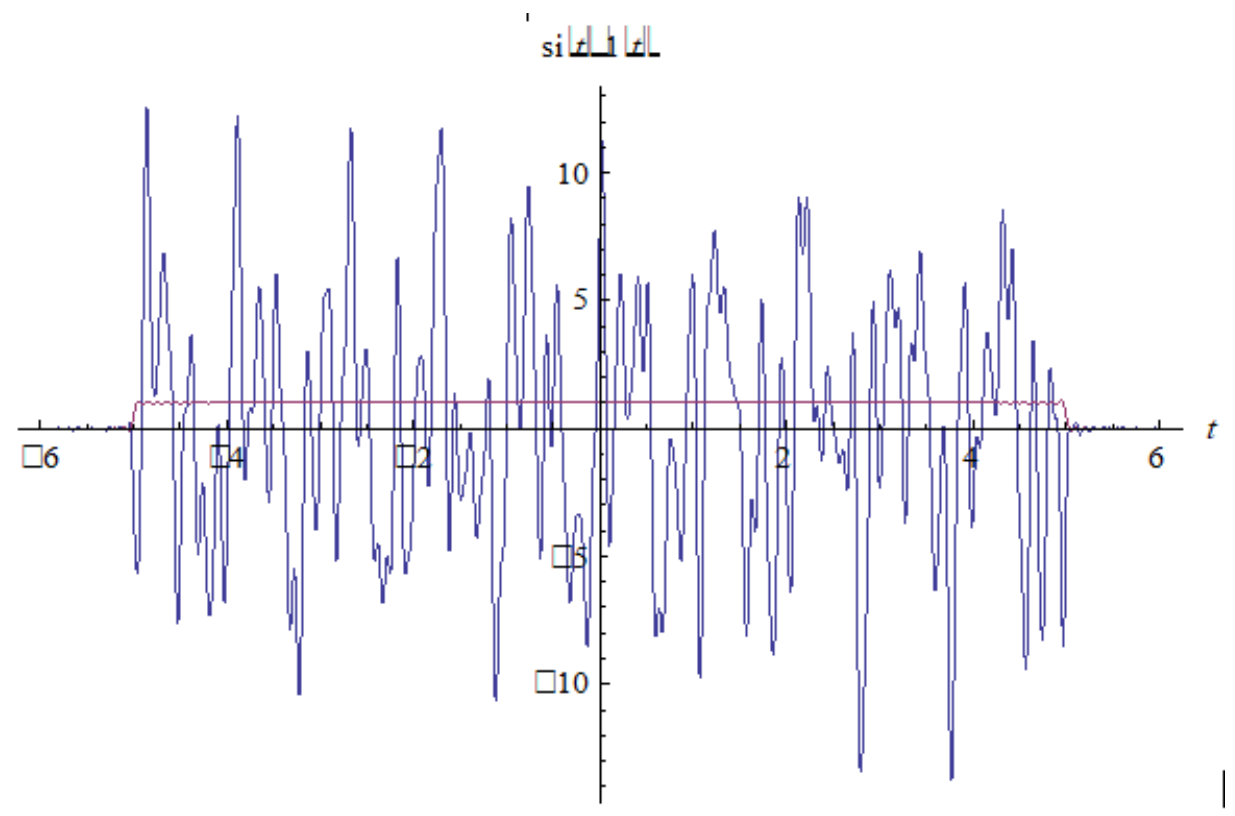


Figure 9. Shannon interpolation error

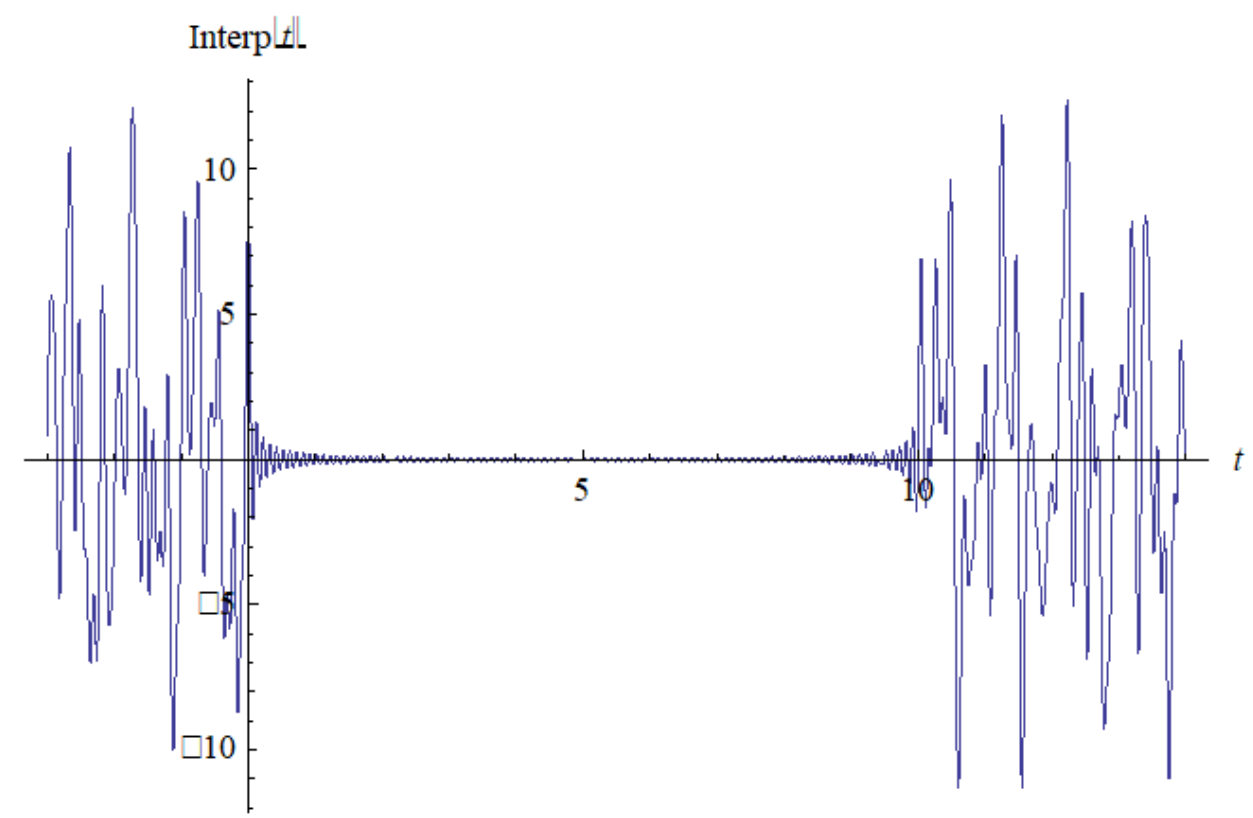

Figure 10. Errors inside the time interval $(0,5)$. without filter

Zoom $\mid$.

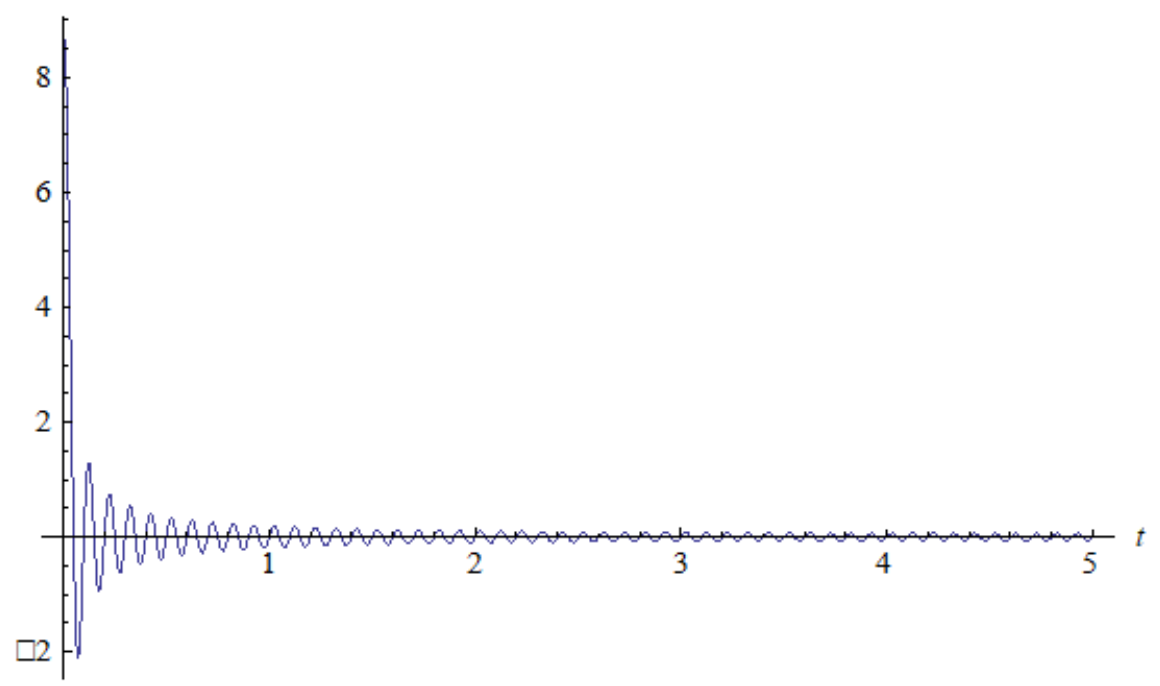


Figure 11. Interpolation errors for Relation 22

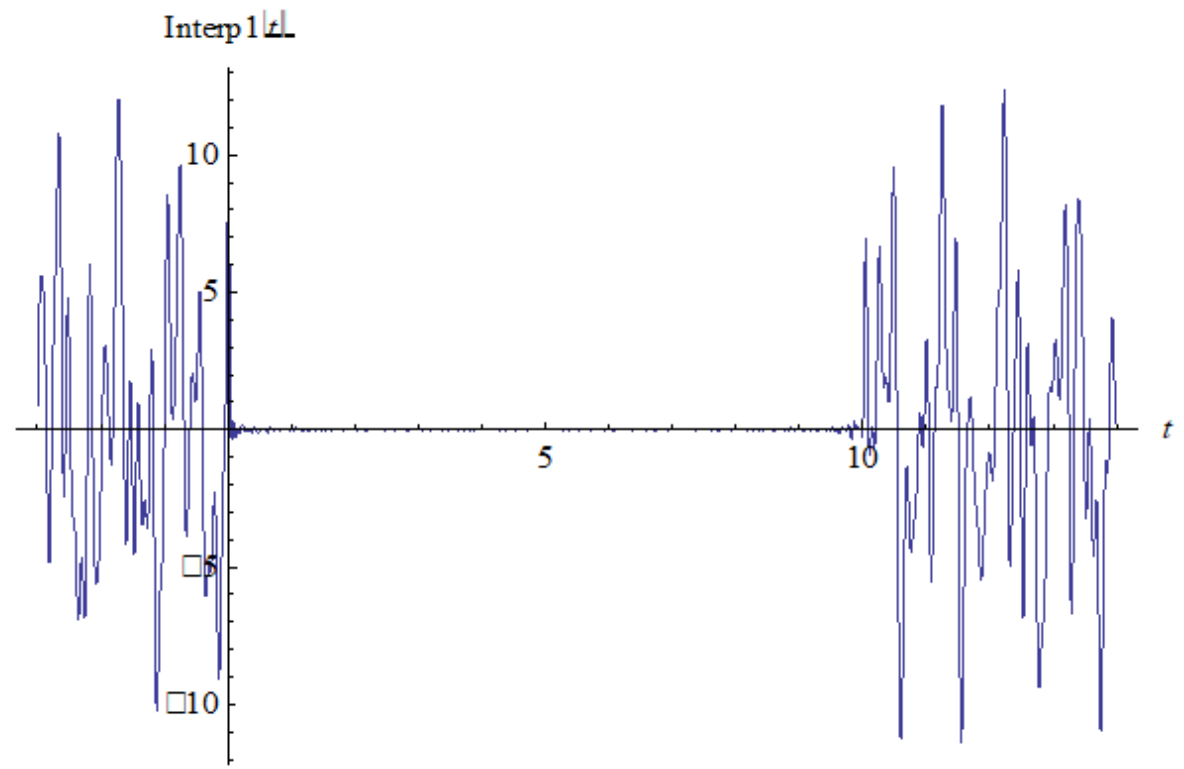

Figure 12. Sampling function and its spectrum.

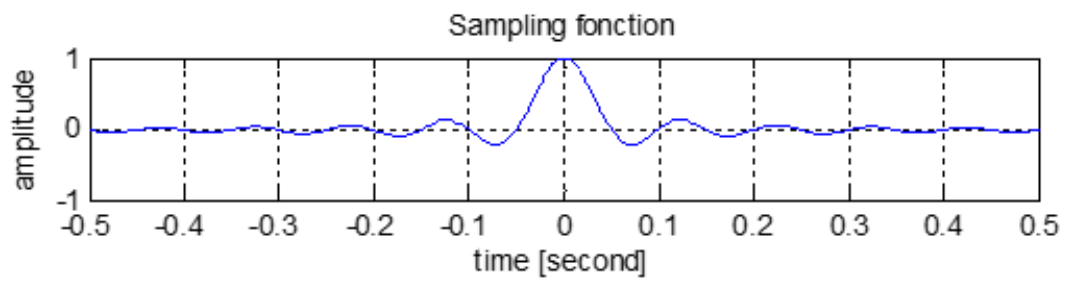

Absolute spectrum of the Sampling fonction

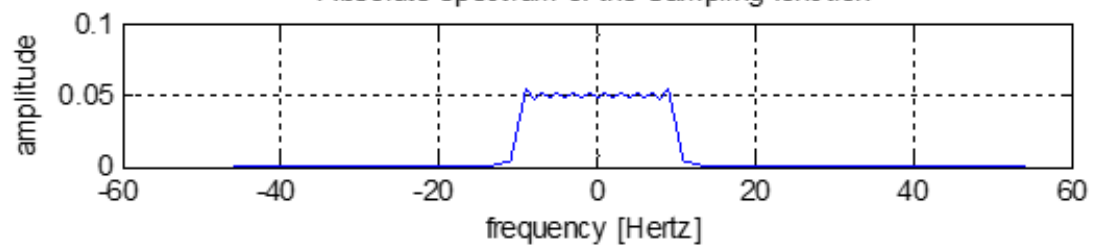

Angle spectrum of the Sampling fonction

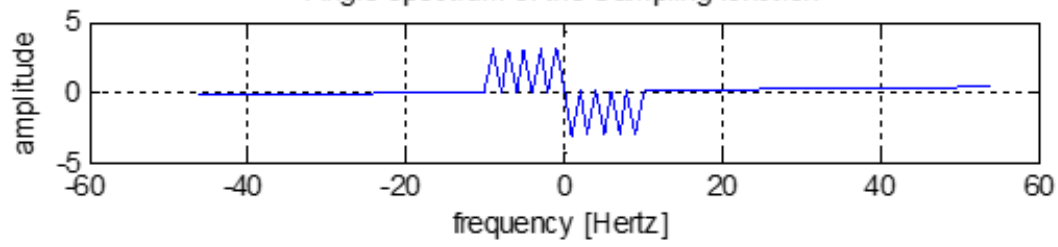


Figure 13. Instantaneous energy and cumsum

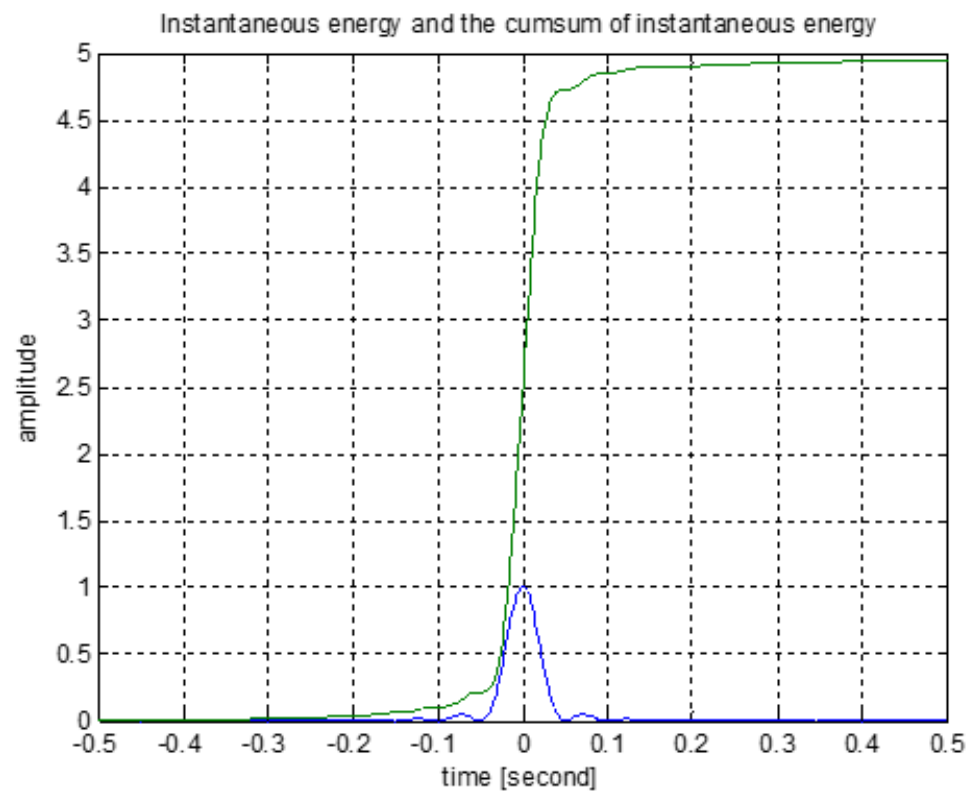

Figure 14. Input signal, its energy and its of instantaneous energy. instantaneous frequency.
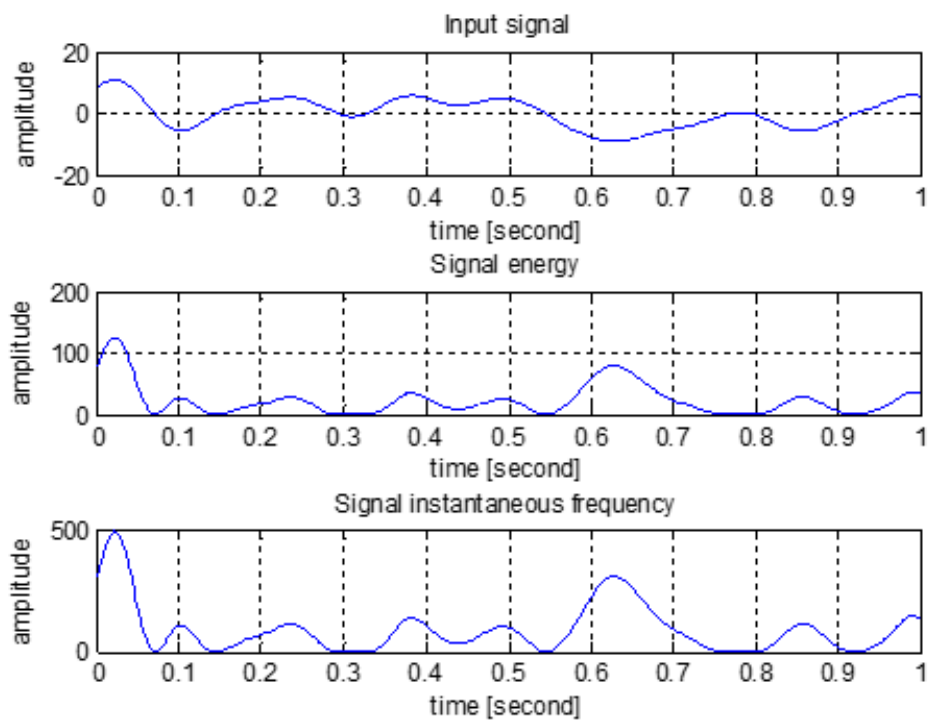
We remember that the interaction between an elementary atomic particle and electromagnetic wave is realized only when the correlation energy between these objects is enough bigger and then, if the frequency of the wave is greater or at list equal to the equivalent frequency of elementary atomic particle.

\section{ABOUT INERTIA IN ROTATION}

Let us consider that a rotating material body, during its rotation, stores energy inside itself by means of elastic deformation of its volume. To keep constant the energy of such system, the sum of the mean value elasticity torques and the other torques due to the movement of rotation must not change. Let us consider a solid matter of revolution that spins around its symmetry axis OZ. Hence, for such object, following Lindsay (Lindsay, R. B, $(1961,1965)$ and using the Euler equations in which we introduce also the equivalent elasticity torques, yields to:

$$
\frac{d}{d t}\left[\left\{\begin{array}{c}
I_{x x} \cdot \dot{\omega}_{x}-I_{x x} \cdot \omega_{y} \cdot \omega_{z}+I_{z z} \cdot S \cdot \omega_{y}+k_{\theta} \cdot \theta=L_{x} ; \\
I_{x x} \cdot \dot{\omega}_{y}+I_{x x} \cdot \omega_{x} \cdot \omega_{z}-I_{z z} \cdot S \cdot \omega_{x}+k_{\psi} \cdot \psi=L_{y} ; \\
I_{z z} \cdot \dot{S}+k_{\xi} \cdot \xi=L_{z}
\end{array}\right\}\right] \equiv 0
$$

and then:

$$
\left\{\begin{array}{c}
I_{x x} \cdot \dot{\omega}_{x}-I_{x x} \cdot \omega_{y} \cdot \omega_{z}+I_{z z} \cdot S \cdot \omega_{y}+k_{\theta} \cdot \theta=0 \\
I_{x x} \cdot \dot{\omega}_{y}+I_{x x} \cdot \omega_{x} \cdot \omega_{z}-I_{z z} \cdot S \cdot \omega_{x}+k_{\psi} \cdot \psi=0 ; \\
I_{z z} \cdot \dot{S}+k_{\xi} \cdot \xi=L_{z}
\end{array}\right\} \equiv \text { constant }
$$

In these equations, $\omega_{x}, \omega_{y}, \omega_{z}$ are (respectively) the angular speeds around the moving axis OX, OY, OZ, $I_{x x}, I_{z z}$ the moments of inertia where, due to the symmetry $I_{x x} \equiv I_{y y}$ and $\dot{\omega}_{x}, \dot{\omega}_{y}, \dot{\omega}_{z}$ are (respectively) the angular accelerations. In relation $S=s+\omega_{z}, s$ is the angular spin. The variables $L_{x}, L_{y}, L_{z}$ are (respectively) the external applied torques where $L_{x}=L_{y}=0$, and where $L_{z}$ is used to keep the spin of the mass nearly constant. The variables $\theta, \psi, \xi$ are the Euler angles and the variables $k_{\theta}, k_{\psi}, k_{\xi}$ are (respectively) the equivalent elasticity coefficients. This is a 3D system of differential equations. From a physical point of view, this is equivalent to saying that such a system may store inside its kinetic inertial energy but this is very well known for the flywheels. The solution of equation (28) leads to ultra-elliptical functions. An advanced analyzes of relation (28) shows that a centrifugal torques change energy with torques on other space dimensions. For example, the term $I_{x x} \cdot \omega_{x} \cdot \omega_{z}$ change the energy between $\mathrm{OX}$ an $\mathrm{OZ}$ axis.

Let now simply considering a well-balanced cylindrical body in rotation on its axis of symmetry where $m$ is the mass of a given point, $r, \theta$ are (respectively) the rotation radius and angle of rotation of this point. The equivalent elasticity coefficient for this mass point is $k_{\theta}$ and we have considered only the deformation of the matter due to the modification of the radius $r$. Starting from relation (28), we may consider a simple mathematical model where any point of this matter in pure rotation satisfies the relation: 
$m . \ddot{\theta}-m \cdot \dot{\theta} \cdot \dot{\varphi}+k_{\theta} \cdot \theta=L ;$

This relation shows that the term $m \cdot \dot{\theta} \cdot \dot{\varphi}$, equivalent to centrifugal torques, transfer energy from $\dot{\theta}$ to $\dot{\varphi}$ and this is not desired for a flywheel and/or for a car wheel as a car wheel with a speed greater than a given angular speed leads to vibrations that may even endanger this car. This implies that for a speed less than an acceptable angular speed, the term $m . \dot{\theta} \cdot \dot{\varphi}$ must be replaced by $m . \dot{\theta}^{2}$ and the relation (29) becomes:

$m . \ddot{\theta}-m \cdot \dot{\theta}^{2}+k_{\theta} \cdot \theta=L$

which is a differential equation.

From physical point of view, any mass point of a body in pure rotation begins with a centrifugal force that elongate the rotation radius of this point. This is due to the fact that the heavier atomic particle, the nuclei, are pushed away from the rotation center by the centrifugal force. The equivalent elasticity of any atom will create a normal force to compensate this elongation. Then, the equivalent instantaneous energy will be:

$m . \ddot{\theta} . \theta-m \cdot \dot{\theta}^{2} . \theta+k_{\theta} \cdot \theta^{2}=E_{R}$

where $m . \ddot{\theta} . \theta+k_{\theta} \cdot \theta^{2}$ is the internal stored inertial energy of the equivalent mass-spring oscillator, $m . \dot{\theta}^{2} . \theta$ is the equivalent energy of centrifugal force and $E_{R}$ is the initial energy that push in rotation this body. As, without any other interactions, the rotation movement energy is conservative, in 'steadystate', the relation (31) becomes:

$m . \ddot{\theta} \cdot \theta+k_{\theta} \cdot \theta^{2}=m \cdot \dot{\theta}^{2} \cdot \theta$

The trajectory of any point ' $i$ ' of this body is $l_{i}=r_{i} \cdot \theta$ and the time-variations of $\theta$ leads to time-variations of $r$. Then from the relation (31) in 'steady-state', the centrifugal force is constant and we may write:

$m . \ddot{r}+k . r=F ;$

where $r$ is the rotation radius and a constant centrifugal force is $F$.

The solution of this differential equation is:

$r=\frac{F}{k}+C_{1} \cdot \operatorname{Cos}\left[\sqrt[2]{\frac{k}{m}} \cdot t\right]+C_{2} \cdot \operatorname{Sin}\left[\sqrt[2]{\frac{k}{m}} \cdot t\right]$

where $C_{1}, C_{2}$ are integration constants. We observe that due to the symmetry of rotation, the force $F$ exerted on each radius is compensated by its equivalent symmetrical radius and also, that the equivalent tangential rotation speed $v_{\tan }=\dot{\theta} . r$ is not constant as $r$ change in time: 
$r=r_{0}+\Delta r=r_{0}+\frac{F}{k}+C_{1} \cdot \operatorname{Cos}\left[\sqrt[2]{\frac{k}{m}} \cdot t\right]+C_{2} \cdot \operatorname{Sin}\left[\sqrt[2]{\frac{k}{m}} \cdot t\right]$

where $r_{0}$ is the radius with $\dot{\theta} \equiv 0$. It can be observed that the mean value of $\Delta r$ is $\Delta \tilde{r}=\frac{F}{k}$.

As this movement is mathematically modeled by trigonometric functions, the angular speed $\dot{\theta}$ will have a 'constant mean value' over the period of these functions.

Function of the external applied energy, such a system may store more energy by increasing its radius $r$. The internal energy of such system is the energy of 3D progressive ultra-acoustic waves. In simulation, figure 15 shows the orbits of the top value of $r$, in blue at a given angular speed, in green at a very low angular speed and in red the mean value of the orbit in blue. Simulation parameters have been chosen to better illustrate the physical model. In simulation, figure 16 shows the function $r[t]$ using the same parameters as in the figure 15 .

The corresponding ('resonant') frequency of the signal from relation (34) is:

$f_{r}=\frac{\sqrt[2]{\frac{k}{m}}}{2 . \pi}$

Let now suppose that an iron molecule from a given matter is in rotation. Accepting that the elasticity coefficient of iron may correspond to its molecule, it results:

$f_{\text {riron }}=\frac{\sqrt[2]{\frac{k}{m}}}{2 . \pi}=\frac{\sqrt[2]{\frac{\sim 10^{4}}{9.2735 \times 10^{-26}}}}{2 . \pi} \cong 5.22635 .10^{13} \mathrm{~Hz}$

This is equivalent to an ultra-acoustic wave and its wave length $\lambda_{\text {iron }}$ will be:

$\lambda_{\text {iron }}=\frac{v_{i r o n}}{f_{r}}=\frac{5130}{5.22635 .10^{13}}=9.81565 .10^{-11} \mathrm{~m} ;$

The radius of a neutral atom is between 0.3 and $3.10-10 \mathrm{~m}$. As a molecule is generally composed of many atoms, the radius of a molecule may be given by radius $_{m}=\alpha_{\text {.radius }}$ atom where the coefficient $\alpha$ depends on the nature of the matter being considered. Then, because $\lambda_{\text {iron }}<$ radius $_{m}$, the acoustic wave being considered can interact directly with the atomic particles that compose this molecule. To keep this rotational movement, the mass has to exchange energy with the environmental gravitational field. This may be done because, due to the ultra-acoustic wave, the polarons (Wikipedia, Polaron, 2015) existing inside the matter will move and then, a gravitational wave may result with the same frequency but with a wavelength $\lambda$ :

$\lambda=\frac{c}{f_{r}}=\frac{3.10^{8}}{5.22635 .10^{13}}=5.74014 .10^{-6} \mathrm{~m} ;$ 
Figure 15. Orbit of the top value of $r$ :

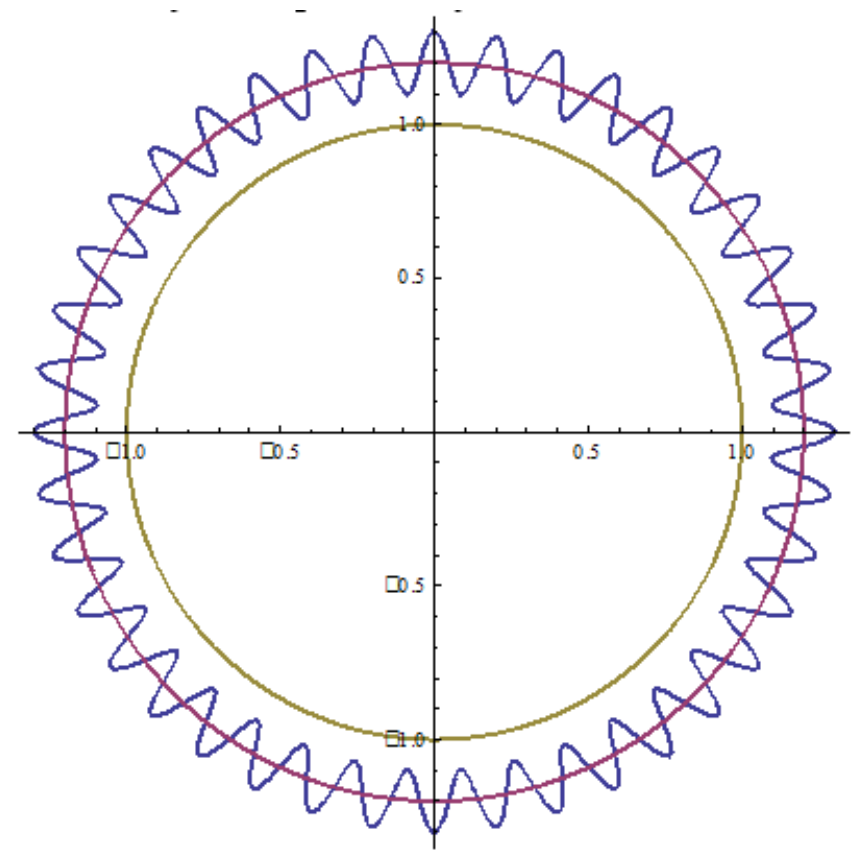

Figure 16. Function $r$.

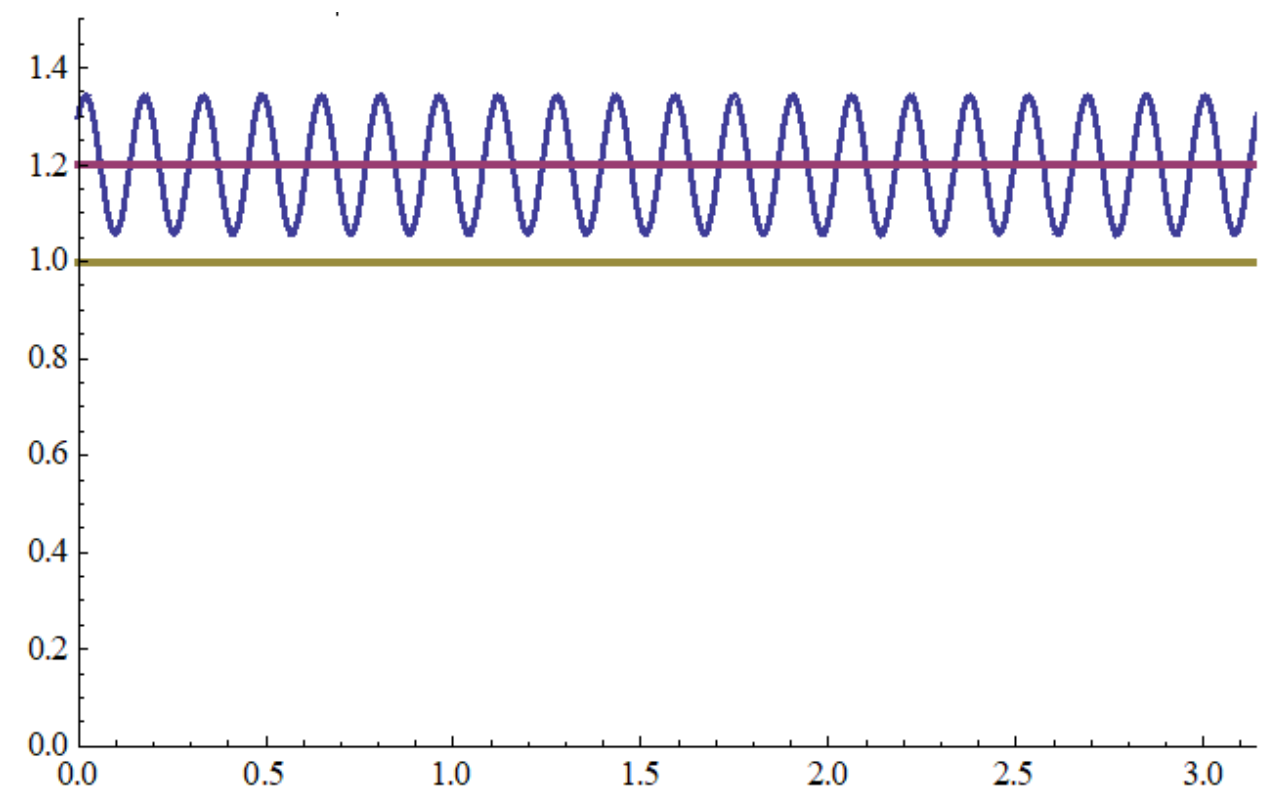


Relation (33) may be considered as equivalent to an electrical LC coil-condenser system. For a LC system, an input signal frequency near or equal to the resonance frequency $f_{r}=\frac{1}{2 \cdot \pi \cdot \sqrt[2]{L \cdot C}}$ leads to increased amplitude of the output signal and eventually to increased electromagnetic wave radiation. Then, a matter in rotation at a high-enough angular speed may be considered as equivalent to a gyroscope and it will transfer energy between its spin, precession and nutation movements but it will also transfer energy to the gravitational field by sending gravitational waves.

A matter in rotation on which no external forces/torques are applied, keeps its movement in space by inertia and then also maintains its rotational energy. This leads to the idea that the ultra-acoustic waves existing inside the matter interacts with the equivalent gravitational waves to ensure the matter rotation in space. It is known that the propagation speed of an ultra-acoustic wave is smaller than the propagation speed of gravitational waves, but their frequencies must be the same to exchange energy. The gravitational and ultra-acoustic wave's lengths are denoted by $\lambda, \lambda_{s}$ and because the frequencies of these waves are the same, it results:

$$
\left\{\begin{array}{c}
\lambda=c . T \\
\lambda_{s}=v_{s} . T
\end{array}\right.
$$

where $T$ is the time-period of these waves, $v_{s}$ is the speed of the acoustic waves, and $c$ is the speed of the light and of the gravitational waves. As this matter is only in rotation, these equivalent ultra-acoustic and gravitational waves may differ at a given time instant only in 'phase' and then, a correlation may exist at each time. In fact, the mechanical energy $E_{r}$ stored by a mass in rotation is given by:

$$
\left\{\begin{array}{l}
E_{r}=\frac{I . \omega^{2}}{2} \\
\vec{v}_{r}=\vec{\omega} \times \vec{r} ;
\end{array}\right.
$$

where $\omega$ is its angular speed, $\vec{v}_{r}$ is the tangential speed, $I$ is the moment of inertia, ' $x$ ' is the vector cross product, and $r$ is the rotation radius.

The diameter of a matter object in rotation increases because of the mean value deformation of some of its molecules. Intuitively, we may consider that the external surface, orthogonal to its diameter in rotation, is dynamically deformed due to the ultra-acoustic inertial wave. Then, by analogy to a 'laser speckle', might this surface be dynamically similar to figure 17 when viewed at very high magnification?

\section{ABOUT INERTIA IN TRANSLATION}

Let now suppose a material body as in figure 18 which flies by inertia in free space on a given direction. The instantaneous energy differential equation that models mathematically this body is: 
Figure 17. Supposed surface dynamically deformation.

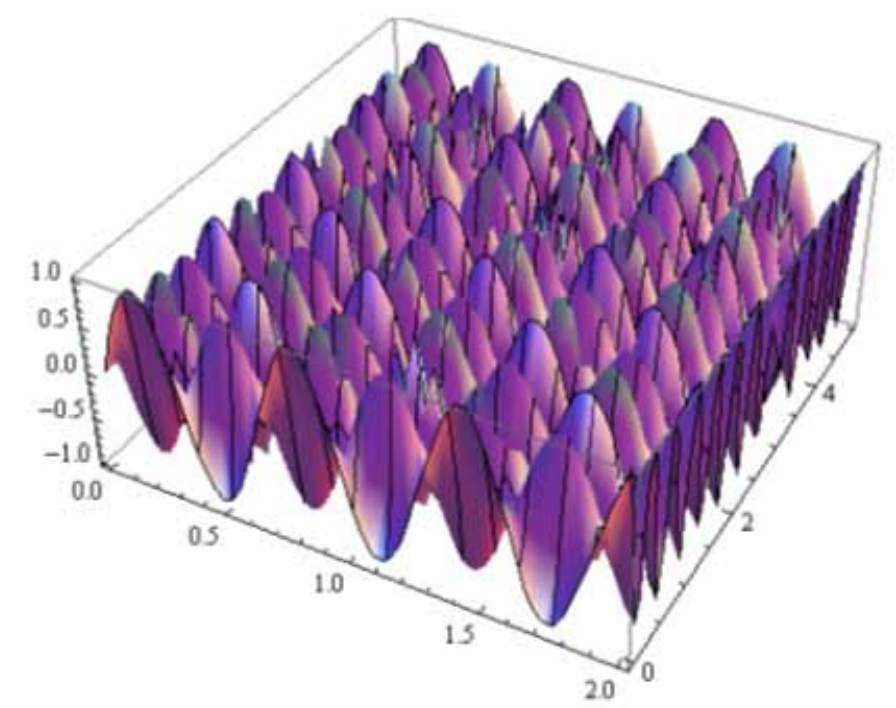

$M \cdot \frac{d^{2} s_{i}(t)}{d t^{2}} \cdot s_{i}(t)+k \cdot s_{i}^{2}(t)=0.5 \cdot M \cdot\left(\frac{d s_{i}(t)}{d t}\right)^{2}=E_{T} ;$

$\Rightarrow M . \ddot{s}_{i}(t) \cdot s_{i}(t)+k \cdot s_{i}^{2}(t)=0.5 \cdot M \cdot \dot{s}_{i}(t)^{2}=E_{T} ;$

where $M$ is the mass of this body, $s_{i}(t)$ is the position in space-time of the center of mass of this rigid body, $k$ is the equivalent elasticity coefficient of this body, $E_{T}$ its total energy, $M . \ddot{s}_{i}(t) . s_{i}(t)$ represent the potential energy, $0.5 . M . \dot{s}_{i}(t)^{2}$ represent the kinetic energy and $k . s_{i}^{2}(t)$ the elastic energy. As for the inertia in rotation, in absence of any other interactions, the mean value of the energy $E_{T}$ does not change. Then:

Figure 18. Body in translation.

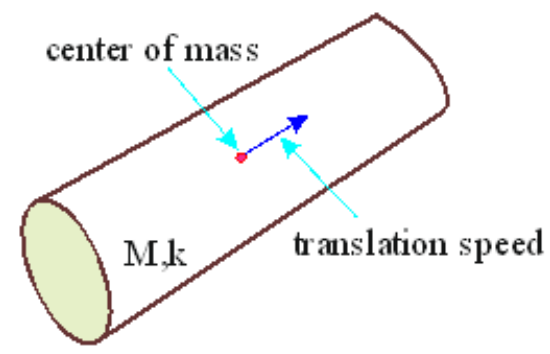

Body in translation 


$$
\begin{aligned}
& \frac{d\left\{M . \ddot{s}_{i}(t) . s_{i}(t)+k \cdot s_{i}^{2}(t)=0.5 \cdot M \cdot \dot{s}_{i}(t)^{2}=E_{T}\right\}}{d t}=0 ; \Rightarrow \\
& M . \ddot{s}_{i}(t) . s_{i}(t)+k \cdot s_{i}^{2}(t)=0.5 \cdot M \cdot \dot{s}_{i}(t)^{2}=E_{T} ;
\end{aligned}
$$

An equivalent equation for forces is obtained if one divides relation (43) by $s_{i}(t)$. It results:

$$
\begin{aligned}
& M . \ddot{s}_{i}(t)+k . s_{i}(t)=\frac{0.5 \cdot M \cdot \dot{s}_{i}(t)^{2}}{s_{i}(t)}=\frac{E_{T}}{s_{i}(t)}=F ; \Rightarrow \\
& M . \ddot{s}_{i}(t)+k . s_{i}(t)=\frac{0.5 \cdot M \cdot \dot{s}_{i}(t)^{2}}{s_{i}(t)}=F ;
\end{aligned}
$$

It may be remarked that the terms $M . \ddot{s}_{i}(t)+k . s_{i}(t)$ are similar to relation (32) that correspond to the ultra-acoustic inertial waves in rotation and the term $\frac{0.5 \cdot M \cdot \dot{s}_{i}(t)^{2}}{s_{i}(t)}$ may be put in conjunction with centrifugal force. In fact, the centrifugal force is mathematically modeled by $\frac{M . \dot{r}_{i}(t)^{2}}{r_{i}(t)}$ where $r_{i}(t)$ is the rotation radius. It can be observed that, excepting the coefficient ' 0.5 ' that does not exists in Einstein energy relation $E=m . c^{2}$, if $r_{i}(t)$ is very big and for short enough distances, the length $l(t)=\theta \cdot r_{i}(t)$ may be considered as $s_{i}(t)$. The solutions of differential equation of relation (42) leads to ultra-elliptic functions. Anyhow, it is known that in free space, a body in translation, without any other interaction, will have a constant mean value speed. Due to the very high frequency of the ultraacoustic internal waves, the mass inertia of a body will easily 'filter the equivalent signal of these waves' so that the mean value of translation speed will be constant. We can remark that the inertia in translation results by interaction between the ultra-acoustic waves existing inside the body in translation and the gravitational field.

From physical point of view, a 'mechanical pushed' material body in free space will have the nuclei of its atoms shift less than the other atom structure because nuclei are the heaviest part of atoms. This 'stores' the elastic energy inside the atoms and start the ultra-acoustic waves as progressive $3 \mathrm{D}$ waves. The resulting kinetic energy move the body in translation. The relation (32) is formally similar to the relation (44). Inertia in translation supposes a mobile that move with a constant mean value speed and have (about) the same inertial ultra-acoustic waves for the whole mobile. The ultraacoustic inertial waves may be considered as an 'inertial polarization of the matter'. On earth, a given item is attracted by the terrestrial gravitation but may also move in any other direction by inertia. Following Einstein, it seems that gravitation polarization is similar to inertial polarization. Gravitation polarization energy is 'pumped' all the time by earth but inertial polarization, only from time to time. For that reason, an earth satellite needs sometimes an external 'rocked engine' to be maintained on its orbit. Mathematically, on earth, the time-variation of the gravitational potential energy is $d E_{P}=d(m . \ddot{x} . x)=m . \ddot{x} . d x$ as $\ddot{x}=g=$ const where $g$ is the terrestrial acceleration. Observing that $m . \ddot{x} . d x=m . \frac{d \dot{x}}{d t} \cdot d x=m . \frac{d x}{d t} \cdot d \dot{x}=m . \dot{x} . d \dot{x}$ it results that $E_{P}=\int m . \dot{x} \cdot d \dot{x}=0.5 \cdot m \cdot \dot{x}^{2}=E_{K}$ where $E_{K}$ is the kinetic energy. We may remark that the time-variation of mechanical impulses energy is $d(m . \dot{x} . x)=m . \ddot{x} . x+m . \dot{x}^{2}$ which leads to potential energy and to kinetic energy apart of the multiplicative coefficient 0.5 . 
Let now consider a given atomic particle belonging to a matter of a body like a Levitron-type gyroscope. If the gravitational 'charge' increases on this atomic particle, the equivalent elliptic modulus $m$ of the Jacobi representation function will increase too. This increases also the angle of nutation and for $m \rightarrow 1$, the nutation periods will be big and/or even very big. Then statistically, over a precession period, the mean value of the direction of the sum of restoring pulses will wobble a lot. If the angle of nutation will be greater than $\frac{\pi}{2}$, the spin of this particle may even change its direction in space. As results, the number of the atomic particles that had their spin oriented in the direction of the matter movement will decrease and the externally-pumped energy will be used only for chaotic oscillations. Mathematically, this seems to happen near $m=1$. Once $m>1$, the evenelliptic Jacobi function $C n[u, m]$ switches to $D n[u, m]$ and the elliptic integral of the first kind function $F_{m>1}[m]$ becomes complex. Then, the energy is now divided between the real and imaginary part of Jacobi functions. It is known that an ideal physical signal may keep its amplitude in time but in reality, due to hyperbolic functions, it becomes complex and then 'dampened' because its energy is changed into heat. On the other hand, it is known that very-high-speed (superluminal), atomic particles ejected by a radioactive material in water generate a blue light due to the Cerenkov effect and such effect may be considered similar to a sonic boom of an acoustic wave. A physical object like a supersonic plane which flies into terrestrial atmosphere needs more energy and creates a special acoustic (inverted) wave, the sonic boom. In both these cases of sound and light, a kind of 'inverse wave' is associated with the mobile.

For a physical material mobile body in space that move by inertia, the inside ultra-acoustic wave will have a speed $v_{s t}<c$ where $c$ is the speed of the light in space. Denoting by $\lambda, \lambda_{s t}$ the gravitational and ultra-acoustic 'waves lengths' that have the same frequencies it results:

$$
\left\{\begin{array}{c}
\lambda=c \cdot T_{t} \\
\lambda_{s t}=v_{s t} \cdot T_{t}
\end{array}\right.
$$

where the time-period of these waves is $T_{t}$. This means that the ultra-acoustic waves inside the mobile mass will be 'retarded in space' versus the equivalent gravitational waves. Generally, the ratio between $c$ and $v_{s}$ is not an entire number and, as for a PLL device, the energy transfer (correlation) between the gravitational and acoustic waves may take place only in a time interval $T_{t}$ inside which $m_{n}$ number of entire periods of gravitational wave correspond exactly to $n_{n}$ number of entire periods of ultra-acoustic wave. Due to 'inertia', the mobile body move in space with a speed $v_{m}$ and then:

$$
T_{t r}=m_{n} \cdot c \cdot T_{t}=n_{n} \cdot\left(v_{s t}+v_{m}\right) \cdot T_{t} ; \Rightarrow \frac{m_{n}}{n_{n}}=\frac{v_{s t}+v_{m}}{c}
$$

In theory and in absence of any other forces, $v_{m}=\sqrt[2]{\frac{2 \cdot E_{T}}{M}}$. To solve the Diophantine equation (46) (Wikipedia (2016), Diophantine equation) we will consider a signal $S[t]$ composed by 2 sine functions of frequencies proportional to $v_{s}+v_{m}$ and $c$ :

$$
S[t]=\operatorname{Sin}\left[2 \cdot \pi \cdot k_{p} . c . t\right]+\operatorname{Sin}\left[2 . \pi \cdot k_{p} \cdot\left(v_{s}+v_{m}\right) . t\right]
$$


where the proportionality factor is $k_{p}$. It can be observed that the signals composing $S[t]$ may be equivalents to the mathematical model of the corresponding gravitational and acoustic waves. From:

$$
\operatorname{Sin}[x]+\operatorname{Sin}[y]=2 \cdot \operatorname{Sin}\left[\frac{x+y}{2}\right] \cdot \operatorname{Cos}\left[\frac{x-y}{2}\right]
$$

where the term $\operatorname{Sin}\left[\frac{x+y}{2}\right]$ may be considered as a 'carrier' and the term $\operatorname{Cos}\left[\frac{x-y}{2}\right]$ as a kind of 'modulation signal'. If $\operatorname{Cos}\left[\frac{x-y}{2}\right]=0 ; \rightarrow x-y=\pi$; and then, the angles $x, y$ differ only in phase at this point. The points where $\operatorname{Cos}\left[\frac{x-y}{2}\right]=0$ delimit the time interval $T_{t}$ because the correlation might be done only between 'phase shifted' signals. For $k_{p} . c=299000$ and $k_{p} \cdot\left(v_{s}+v_{m}\right)=21$, the signal $S[t]$ is shown in figure 19. It can be observed that the time interval $T_{t}$ depends also on the ratio of the equivalent speeds $c, v_{s}+v_{m}$ and that the interaction between the gravitational and ultra-acoustic waves 'tries' to diminish the value of $T_{t}$ by adjusting conveniently the inertial speed $v_{m}$. In fact, if the ratio between the equivalent speeds $c, v_{s}+v_{m}$ is irrational and/ or transcendent, $T_{t} \rightarrow \infty$. That happened, as example, when $v_{s}+v_{m} \rightarrow c$ and in this case, the correlation between the gravitational and ultra-acoustic waves cannot take place. It is also interesting to observe that scientists from Eindhoven, Netherlands, have discover a new polymer that 'walks' when illuminated (Gelebart et al., 2017). May we consider this as being like a kind of 'tractor ray' where all the necessary energy of 'walking' is brought only by an electromagnetic wave?

\section{ABOUT WAVES AND SPACE DIMENSIONS}

It is known that the integral of the function cosine leads to the orthogonal sine function and:

$$
\operatorname{Cos}[t]+j \cdot \operatorname{Sin}[t]=e^{j . t}
$$

This generates a circle of radius equal to one in complex plane. If we consider the sine and cosine functions as being statistical distributions, their integrals correspond with the cumulative distribution functions, Similarly, for the Jacobi elliptic functions, one may consider the following relation:

$$
u=F[\Phi, m]=\int_{0}^{\Phi} \frac{d t}{\sqrt[2]{1-m \cdot \operatorname{Sin}^{2}[t]}} ; \Rightarrow \Phi=A m[u, m]
$$

where the modulus of elliptical functions is $m$. Then, the double-periodic functions $C n[u, m], S n[u, m], D n[u, m]$ are defined by: 
Figure 19. Representation of signal $S[t]$.

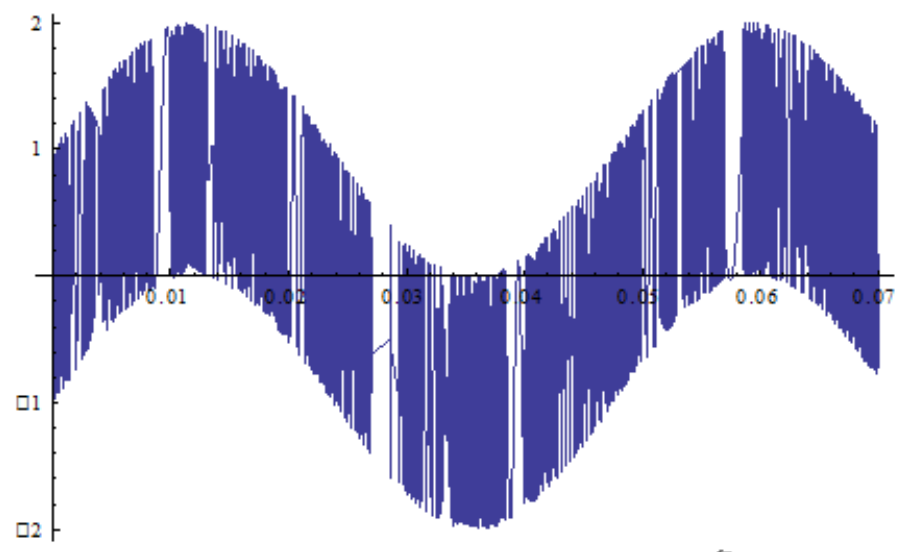

Figure 20. 3D Parametric plot of the integrals of Jacobi functions for $m=0.8$

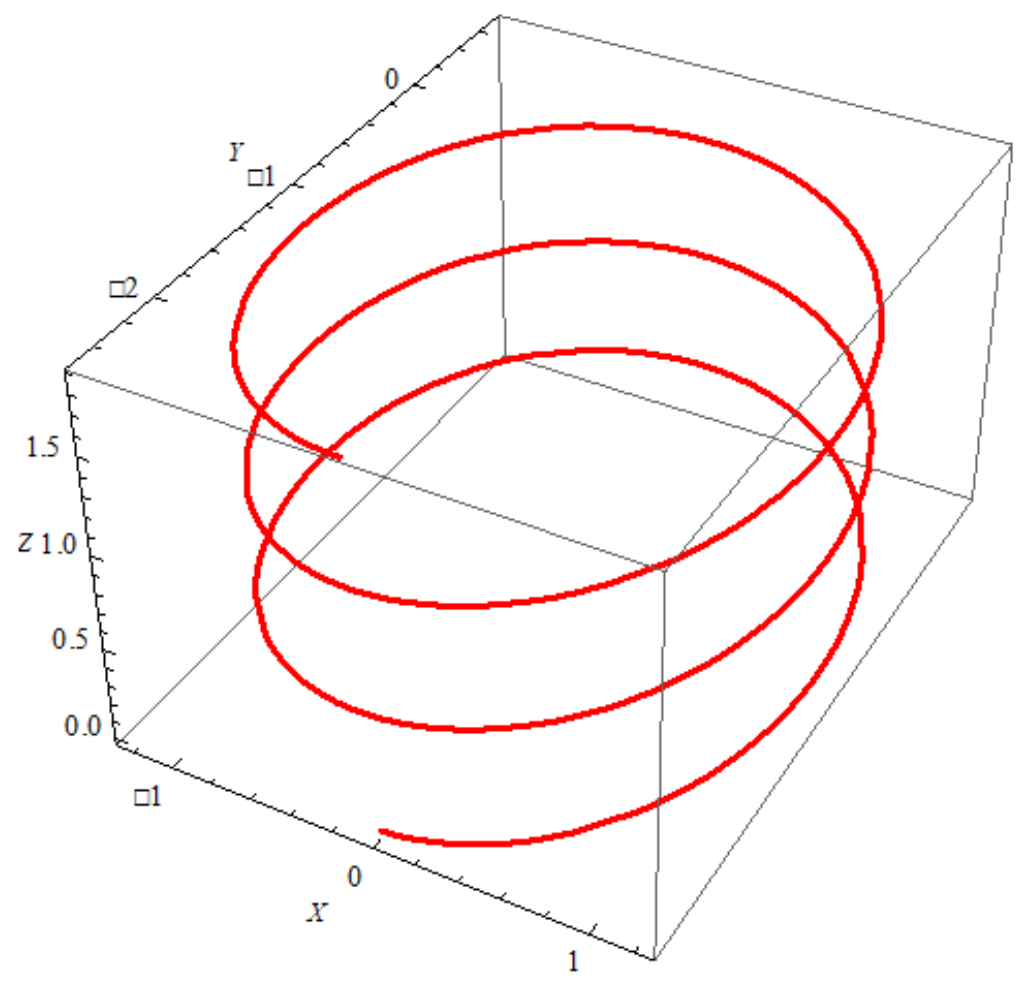




$$
\left\{\begin{array}{c}
C n[u, m]=\operatorname{Cos}[A m[u, m]] \\
S n[u, m]=\operatorname{Sin}[A m[u, m]] \\
D n[u, m]=\sqrt[2]{1-m \cdot S n^{2}[u, m]}
\end{array}\right.
$$

From here and for $m \leq 1$, we may consider a 3D extension in space like:

$$
\left\{\begin{array}{c}
X \Rightarrow \int C n[u, m] \cdot d u=\frac{\operatorname{Arc} \operatorname{Cos}[D n[u, m]] \cdot S n[u, m]}{\sqrt[2]{1-D n^{2}[u, m]}} \\
Y \Rightarrow \int S n[u, m] \cdot d u=\frac{\log [-\sqrt[2]{m} \cdot C n[u, m]+D n[u, m]]}{\sqrt[2]{m}} \\
Z \Rightarrow \int D n[u, m] \cdot d u=A m[u, m]
\end{array}\right.
$$

where $A m[u, m]$ is a Jacobi amplitude function, $u$ is a complex variable, and $m$ the elliptic modulus of these functions. The function $D n[u, m]$ is also called $\Delta A m[u, m]$. The real part of these Jacobi functions for a real part of the variable $u$ generates a spiral, as in figure 20. This curve may be considered as being a '3D extension of a 2D circle.' A nearly similar spiral may be built using trigonometric functions and polynemes as in equation (52). This is shown in figure 21 and in this case, $Z_{t} \Rightarrow \alpha . t$.

$$
\left\{\begin{aligned}
X_{t} & \Rightarrow \operatorname{Cos}[t] \\
Y_{t} & \Rightarrow \operatorname{Sin}[t] \\
Z_{t} & \Rightarrow 0.1 . t
\end{aligned}\right.
$$

One may also observe that for:

$$
m \equiv 0 ;->\left\{\begin{array}{c}
D n[u, 0]=1 \\
S n[u, 0]=\operatorname{Sin}[u] \\
C n[u, 0]=\operatorname{Cos}[u]
\end{array}\right.
$$

and the Jacobi elliptic functions become complex exponential functions and for a real variable $t=\operatorname{Re}[u]$, relation (54) leads to trigonometric functions and:

$$
\int D n[t, 0] \cdot d t=\int d t=t+c o n s t
$$

Relation (55) reconstructs an initial real continuous variable (the time) that has been extended in complex and 'periodized in 3D' with the exception of an unknown constant. Then, the 'initial origin of this real, continuous variable $t$ has been lost'. It seems that at any instant in time, only the transformations with big enough local probabilities may have a chance to be realized. This is in accordance with the 'entropy' of a system and implies that actually, the realization of a transformation 
Figure 21. 3D Parametric plot using trigonometric functions

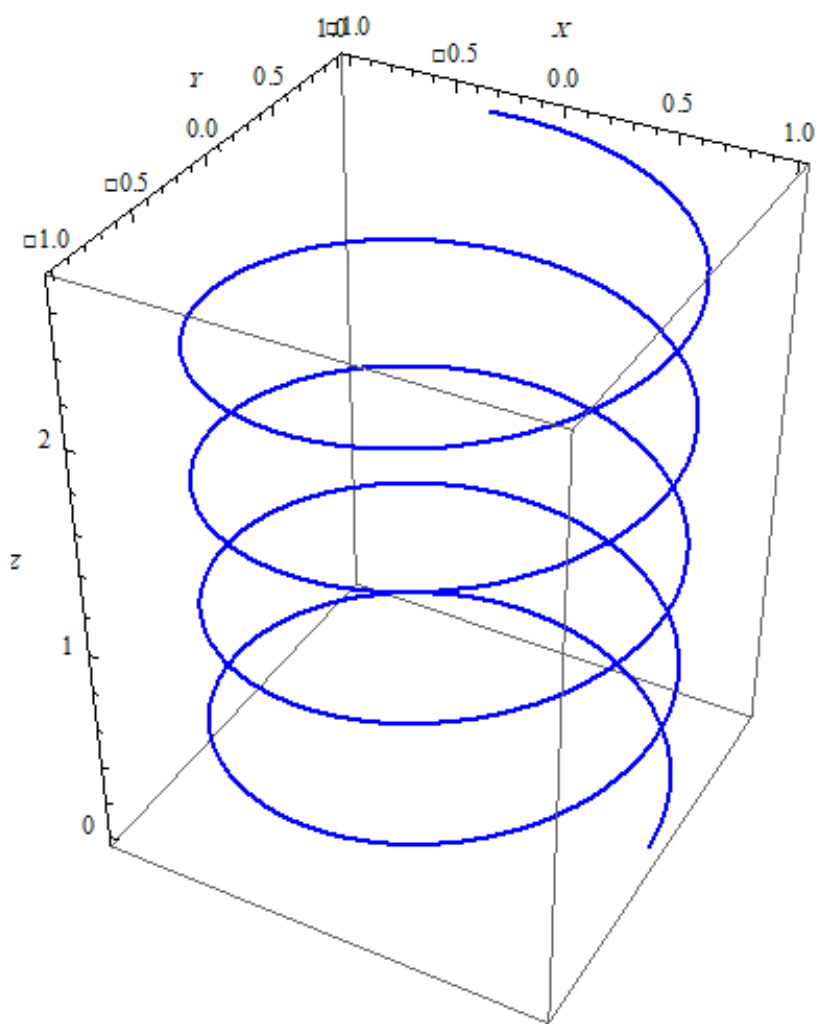

Figure 22. $\operatorname{Re}[A m[u, 0.8]]$ for $0<m<1$ in red and the physical time ( $m \equiv 0$ ) in blue

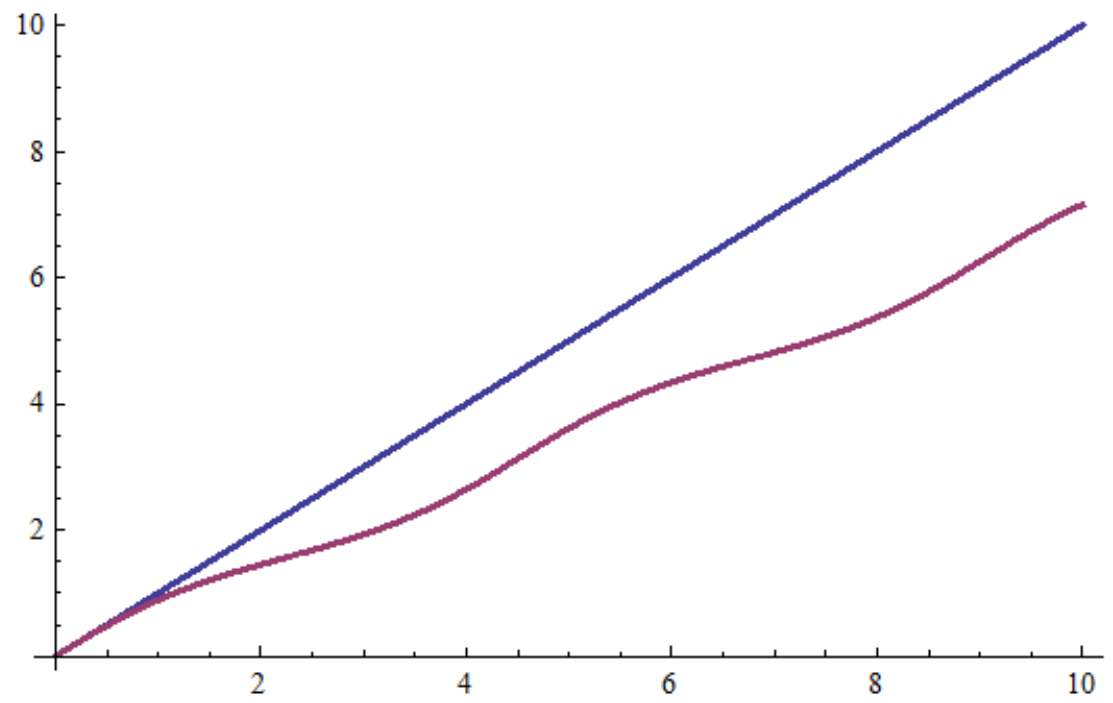


Figure 23. 3D Parametric plot of the integra of Dn[.] Jacobi functions for $m=1.2$

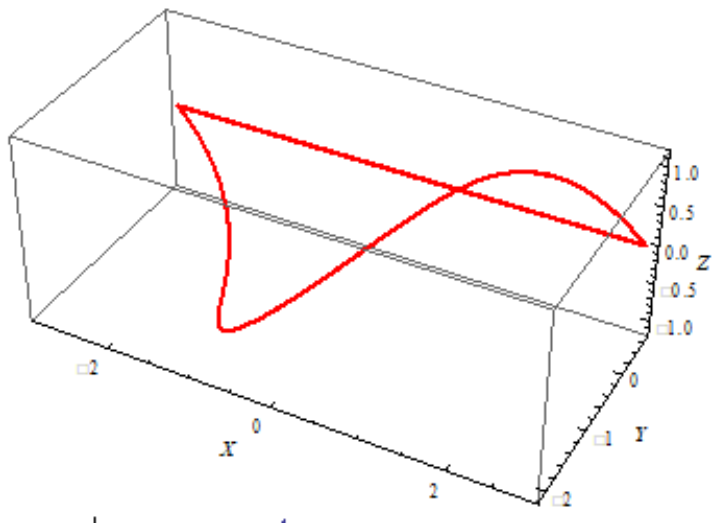

with a small probability needs much energy to be realized. From here, an initial real continuous variable of time that has been extended in complex and 'periodized in 3D', has a very small probability of being reconstructed. It may be reconstructed only in the future like, for example, an audio and/or video recording realized in the past that may be played again only in the future. It may also be observed that $\operatorname{Re}[A m[u, m]]$ for $1 \leq m \geq 0$ is (nearly) like a 'phase function' and that it is different from the physical time. This is presented in figure 22. For $m \geq 1$, relation (55) does no more holds and that can be seen in figure 23 where the movement is periodic and implies only a 'region of the ' $\mathrm{Z}$ ' axis'. It seems that this kind of movement 'stocke locally energy' which may corresponds to the 'increase of the mass of the mobile'. For $m>1$, the function Dn[.]switch to the function $\mathrm{Cn}[$.$] and taking into$ account the relation (52), we may consider a $3 \mathrm{D}$ extension in space like:

Figure 24. $\operatorname{Re}\left[\int C n[u, 1.2] . d u\right]$ for $m=1.2$

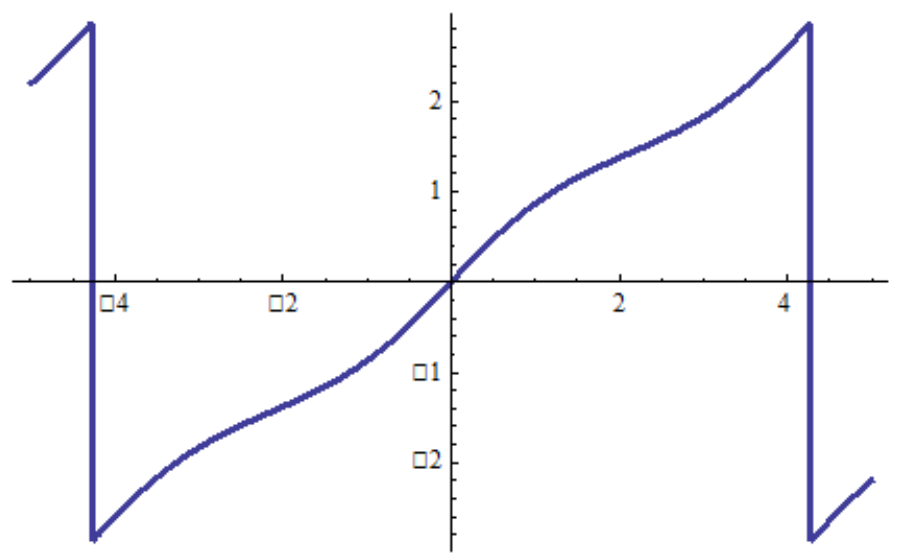


Figure 25. Equivalent 'phase function' of integrals of $\int C n[u, 1.2] . d u$ for $m=1.2$

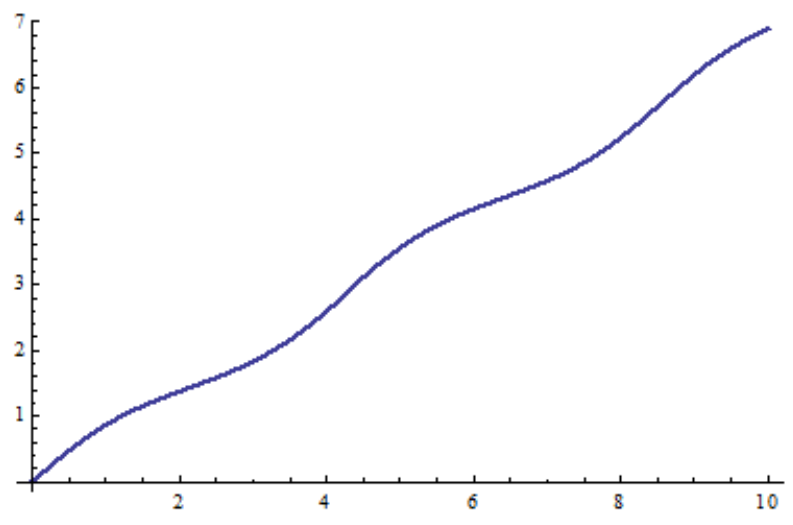

$$
\left\{\begin{array}{c}
X \Rightarrow \int \operatorname{Dn}[u, m] \cdot d u=\operatorname{JacobiAm}[u, m] \\
Y \Rightarrow \int S n[u, m] \cdot d u=\frac{\log [-\sqrt[2]{m} \cdot \operatorname{JacobiCn}[u, m] . J a c o b i D n[u, m]}{\sqrt[2]{m}} \\
Z \Rightarrow \int C n[u, 1.2] . d u=\frac{\operatorname{Arc}[\operatorname{Cos}[\operatorname{Dn}[u, m]]] . S n[u, m]}{\sqrt[2]{1-D n^{2}[u, m]}}+\operatorname{Ladder}[u]
\end{array}\right.
$$

as shown in Figure 24. This figure represents a 'periodic phase function' which, using the Ladder function, may be transformed into a

One can observe that for $m=1.2$ :

$$
\int C n[u, 1.2] \cdot d u=\frac{\operatorname{Arc}[\operatorname{Cos}[\operatorname{Dn}[u, 1.2]]] \cdot \operatorname{Sn}[u, 1.2]}{\sqrt[2]{1-D n^{2}[u, 1.2]}}
$$

as shown in figure 24. This figure represents a 'periodic phase function' which, using the Ladder function (Ciulin, 2010b), may be transformed into a non-periodic phase function as shown in figure 25. It may be also observed that the 'phase functions' obtained for $m<1$ and $m>1$ (as represented in figures 22 and 25) are similar and that the Ladder function is defined as:

$$
\text { Ladder }[t]=\sum_{n=0}^{n \rightarrow \infty} 0.5 . A .\left(1+\frac{t-n . T}{|t-n . T|}\right)
$$

where $A$ is the amplitude of the function $\int C n[u, 1.2] . d u$ and $T$ is its period.

A 3D parametric plot of integrals of Jacobi functions for $m=1.2$ is shown in figure 26. A good similarity with figure 20 may be observed. More, in a 3D parametric plots, the functions for the $\mathrm{X}$ 


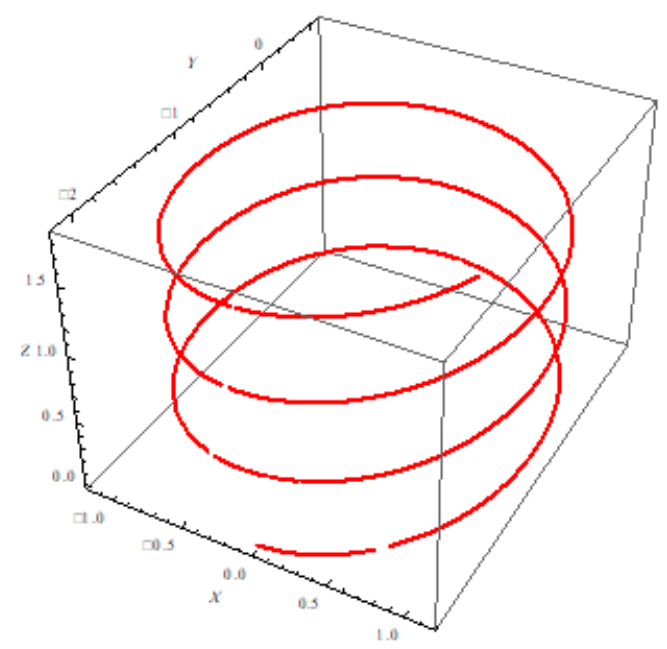

and Y axes, $\int C n[u, m] . d u$, may be replaced with $C n[u, m]$ and $\int S n[u, m] . d u$ by $S n[u, m]$ with nearly similar results.It may be remarked that:

$$
\left\{\begin{array}{c}
C n[u, m]=\sqrt[2]{1-S n^{2}[u, m]} ; \\
D n[u, m]=\sqrt[2]{1-m \cdot S n^{2}[u, m]}
\end{array}\right.
$$

and both even Jacobi elliptic functions $C n[u, m], D n[u, m]$ are defined through an odd Jacobi elliptic function, $S n[u, m]$.

The elliptic integral of the first kind $F[m]$ for $m \leq 1$ and $m \geq 1$ gives the relation between the elliptic module and the quart of periods of the equivalent elliptic functions:

$$
\left\{\begin{array}{c}
F_{m \leq 1}[m]=\int_{0}^{\frac{\pi}{2}} \frac{d \phi}{\sqrt[2]{1-m \cdot \operatorname{Sin}^{2}[\phi]}} ; \\
F_{m>1}[m]=\frac{1}{\sqrt[2]{m} \cdot F\left[\frac{1}{\sqrt[2]{m}}\right]} ;
\end{array}\right.
$$

In fact, for $m \equiv 0$, the $2 \mathrm{D}$ trigonometric functions have as period $2^{2} \cdot \frac{\pi}{2}=2 . \pi$. The absolute (blue) and imaginary (red) parts of the function $F[m]$ are represented in figure 27.

For $m>1$ and then after the pole corresponding to $m \equiv 1$, the even elliptic Jacobi function $C n[u, m]$ becomes like the function $D n[u, m]$ for $m<1$ and the function $F_{m>1}[m]$ becomes complex. Such kind of 'switch' may be physically considered as being equivalent to a special 'rotation in 3D.' This may be placed in conjunction with the fact that for $m>1$, the speed of the equivalent mass increases. For a simple pendulum, the elliptic module $m$ of the Jacobi functions is given by: 
Figure 27. Absolute and imaginary part of $F[m]$ function.

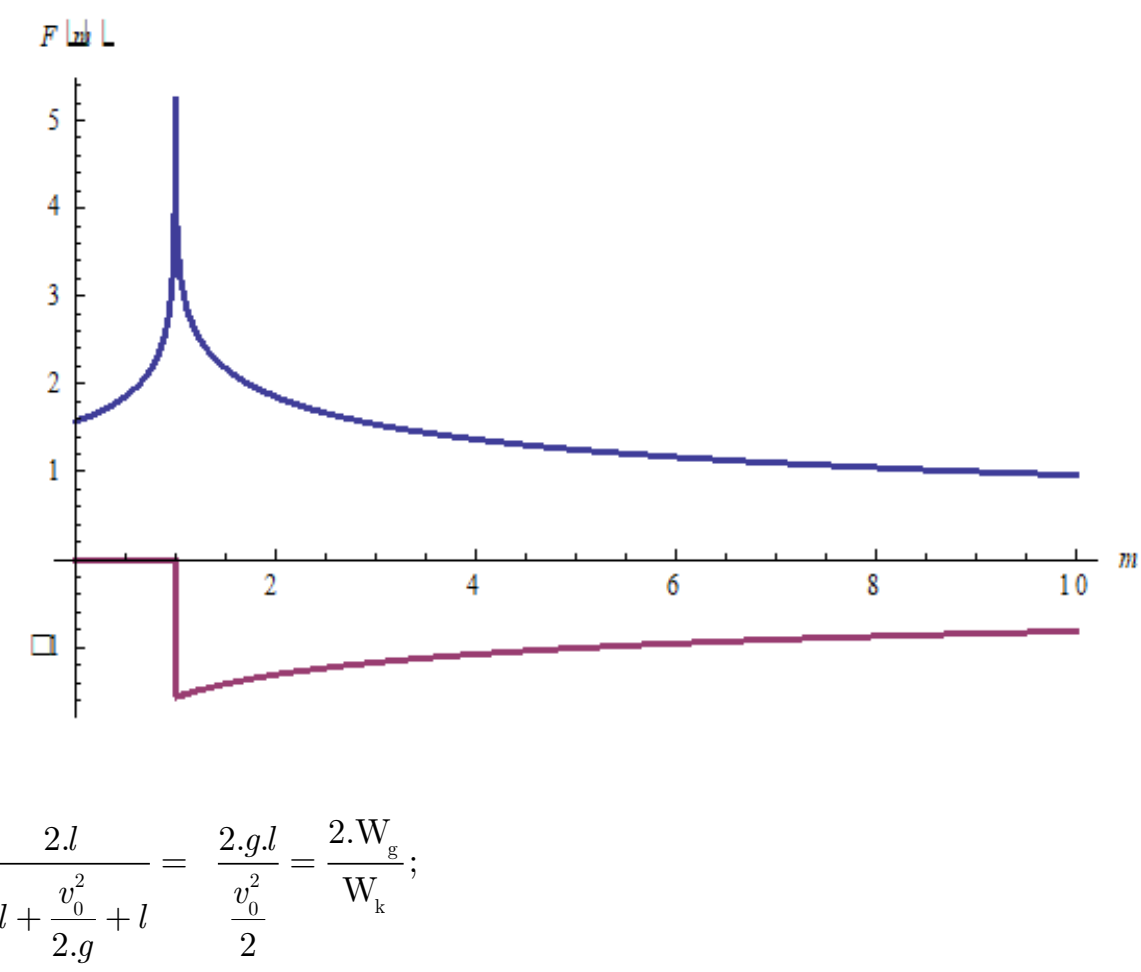

where $l$ is the radius of the trajectory of the mass of the pendulum, $g$ is the earth acceleration of gravitation, and $v_{0}$ is the speed of the mass of the pendulum at the lowest point of its trajectory. It can be remarked that the value of $m$ may be represented by the ratio between twice the gravitational energy $\mathrm{W}_{\mathrm{g}}$ and the kinetic energy $\mathrm{W}_{\mathrm{k}}$ of the pendulum. Then, for very small kinetic energy $\mathrm{W}_{\mathrm{k}}$ of the pendulum, the elliptic module $m$ of the Jacobi functions may tend towards infinity.

Let now consider the hodograph of an associated wave of an electron inside of a magnetron tube. This may be shown in figure 28 as hodograph and in figure 29 as 'trace' of the trajectory of this electron. For simplicity, these parametric representations have been realized using only trigonometric functions. The only difference between the mathematical representation of figures 28 and 29 is that two independent variables $(\mathrm{u}, \mathrm{v})$ have been used for figure 28 but only one (u) for figure 29. In fact, a single independent variable 'draw lines' but two variables 'draw surfaces' in 3D.

As for a Levitron-type gyroscope, some atomic particles may also have as mathematical model the system (Ciulin, D., 2013a, January-March):

$$
\left\{\begin{array}{c}
\left.\left(I_{x x}+\frac{\partial B_{g}}{\partial \theta}\right) \cdot \dot{\omega}_{x}-\left(I_{x x}+\left(\frac{\partial B_{g}}{\partial \psi}\right)^{2} \cdot \frac{1}{\operatorname{Sin}(\theta) \cdot \operatorname{Cos}(\theta)}\right) \cdot \omega_{y} \cdot \omega_{z}+I_{z z} \cdot S \cdot \omega_{y}+\left(\frac{\partial B_{g}}{\partial \psi}\right)^{2} \cdot \frac{\omega_{y} \cdot \omega_{z}}{\operatorname{Sin}(\theta) \cdot \operatorname{Cos}(\theta)}+\frac{\partial B_{g}}{\partial \psi} \frac{s \cdot \omega_{y}}{\operatorname{Sin}(\theta)}\right)=L_{x} \\
\left(I_{x x}+\frac{\partial B_{g}}{\partial \psi} \cdot \frac{1}{\operatorname{Sin}(\theta)}\right) \cdot \dot{\omega}_{y}+\left(I_{x x}+2 \cdot \frac{\partial^{2} \vec{B}_{g}}{\partial \theta, \partial \psi} \cdot \frac{1_{x}}{\operatorname{Cos}(\theta)}\right) \cdot \omega_{x} \cdot \omega_{z}-\left(I_{z z} \cdot S \cdot \omega_{x}+2 \cdot\left(\frac{\partial^{2} \vec{B}_{g}}{\partial \theta, \partial t} \cdot \frac{\omega_{x} \cdot \omega_{z}}{\operatorname{Cos}(\theta)}+\frac{\partial B_{g}}{\partial \psi} \frac{s \cdot \omega_{x}}{\operatorname{Sin}(\theta)}\right)\right)=L_{y} \\
\left(I_{z z}+\frac{\partial \vec{B}_{g}}{\partial \psi} \cdot \frac{1}{\operatorname{Cos}(\theta)}\right) \cdot \dot{S}_{z}=L_{z} ;
\end{array}\right.
$$


Figure 28. Hodograph of the the associated wave.

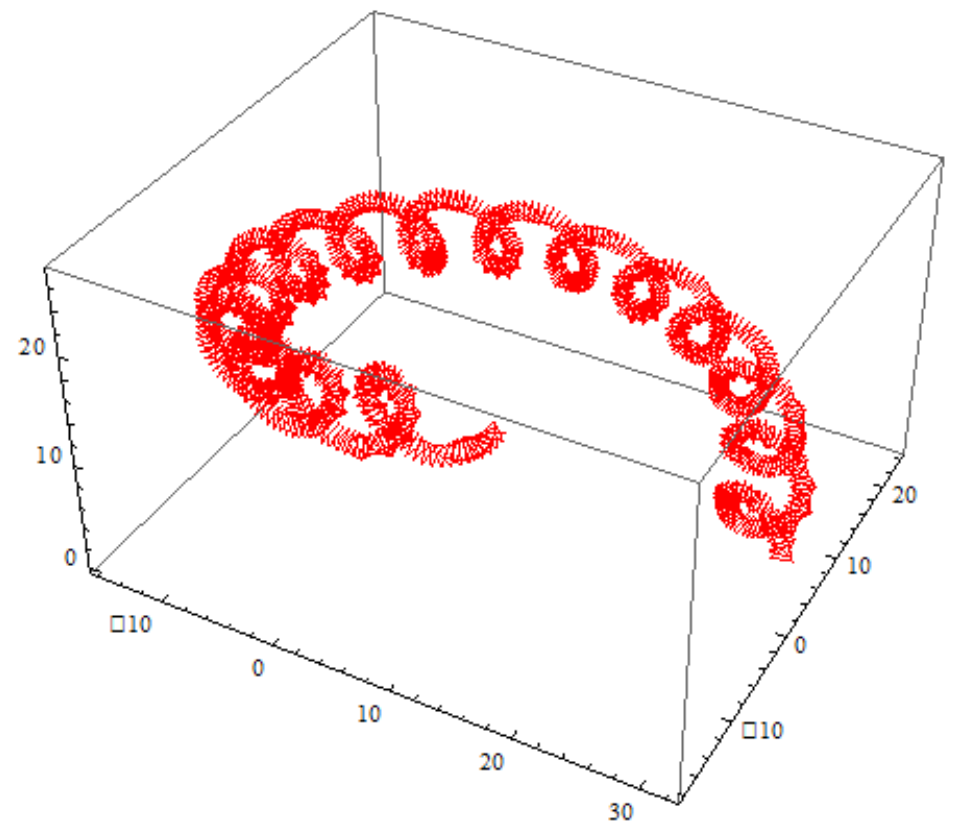

where $L_{x}, L_{y}, L_{z}$ are the (external mechanical) applied torques on the ox, oy, and oz axes, $I_{x x}, I_{y y}, I_{z x}$ are the moments of inertia, $\omega_{z}+s=S$ where $\omega_{z}$ is the angular speed around the oz axis, $s$ is the spin and due to the symmetry, $I_{x x} \equiv I_{y y}$. The angle $\theta$ is connected with the movement of nutation and the angle $\psi$ is connected with the precession movement. The terms $\frac{\partial B_{g}}{\partial(.)}$ and $\frac{\partial^{2} B_{g_{. .}}}{\partial(., .)}$

Figure 29. 'Trace' of an electron.

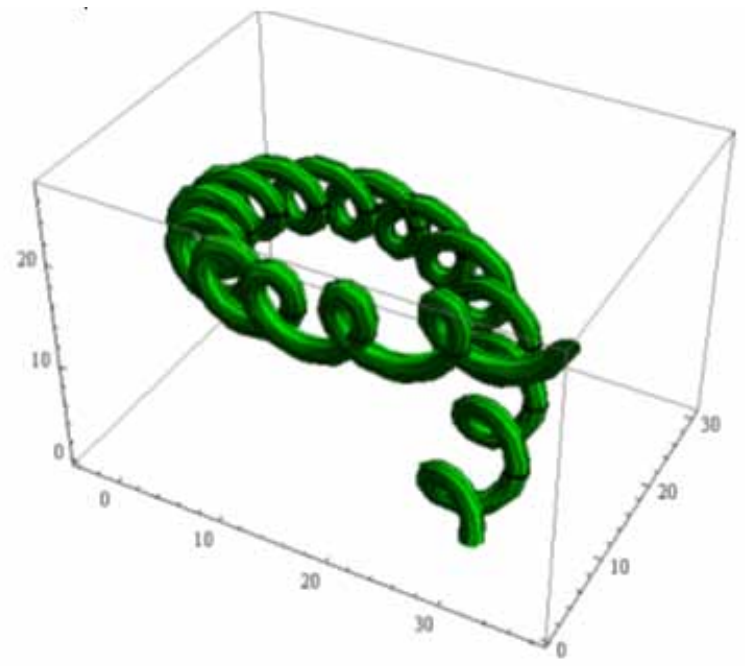


represents the first and second order 'sensitivities' of the variations in time of the magnetic field $\vec{B}_{g}$ due to the variation of the angles $\theta, \psi$. Relation (62) includes the magnetic field $\vec{B}_{g}$, its equivalent 'energy' and supposes that $s$ is nearly constant. This allows to the possibility of transferring energy by means of an electromagnetic field and then ensures the possibility of realizing the necessary bending torques without the necessity of having a 'strong point' in free space.

A close analyze of relation (62) suggest that all the moments of inertia may also be considered as being sensitivities of the gyroscope against the gravitational field $G$ and magnetic field B and that the external applied torque $L_{\theta}, L_{\varphi}, L_{\psi}$ might be also gravitational or electromagnetic. Observing that in nature, any material body is in movement, it seems that it interacts at any time with the gravitational field and also, more or less, with the electromagnetic field. Then, the external applied torque $L_{\theta}, L_{\varphi}, L_{\psi}$ may be considered as interaction torques with gravitational/inertial and/or electromagnetic fields.Let now consider a generalized field:

$U=U[\theta(t), \varphi(t), \psi(t), B[\theta, \varphi, \psi], t]$

that includes the gravitational and electromagnetic fields. From here, it results that:

$$
\left\{\begin{aligned}
d U= & \frac{\partial U}{\partial \theta} \cdot \frac{d \theta}{d t}+\frac{\partial U}{\partial \varphi} \cdot \frac{d \varphi}{d t}+\frac{\partial U}{\partial \psi} \cdot \frac{d \psi}{d t}+\frac{\partial U}{\partial B} \cdot \frac{\partial B}{\partial \theta} \cdot \frac{d \theta}{d t}+\frac{\partial U}{\partial B} \cdot \frac{\partial B}{\partial \varphi} \cdot \frac{d \varphi}{d t}+\frac{\partial U}{\partial B} \cdot \frac{\partial B}{\partial \psi} \cdot \frac{d \psi}{d t}+\frac{\partial U}{\partial t} \\
& d^{2} U=\frac{\partial^{2} U}{\partial \theta^{2}} \cdot \frac{d \theta}{d t}+\frac{\partial U}{\partial \theta} \cdot \frac{d^{2} \theta}{d t^{2}}+\frac{\partial^{2} U}{\partial \varphi^{2}} \cdot \frac{d \varphi}{d t}+\frac{\partial U}{\partial \varphi} \cdot \frac{d^{2} \varphi}{d t^{2}}+\frac{\partial^{2} U}{\partial \psi^{2}} \cdot \frac{d \psi}{d t}+\frac{\partial U}{\partial \psi} \cdot \frac{d^{2} \psi}{d t^{2}}+ \\
& \frac{\partial^{2} U}{\partial B^{2}} \cdot \frac{\partial B}{\partial \theta} \cdot \frac{d \theta}{d t}+\frac{\partial U}{\partial B} \cdot \frac{\partial^{2} B}{\partial \theta^{2}} \cdot \frac{d \theta}{d t}+\frac{\partial U}{\partial B} \cdot \frac{\partial B}{\partial \theta} \cdot \frac{d^{2} \theta}{d t}+ \\
& \frac{\partial^{2} U}{\partial B^{2}} \cdot \frac{\partial B}{\partial \varphi} \cdot \frac{d \varphi}{d t}+\frac{\partial U}{\partial B} \cdot \frac{\partial^{2} B}{\partial \varphi^{2}} \cdot \frac{d \varphi}{d t}+\frac{\partial U}{\partial B} \cdot \frac{\partial B}{\partial \varphi} \cdot \frac{d^{2} \varphi}{d t^{2}}+ \\
& \frac{\partial^{2} U}{\partial B^{2}} \cdot \frac{\partial B}{\partial \psi} \cdot \frac{d \psi}{d t}+\frac{\partial U}{\partial B} \cdot \frac{\partial^{2} B}{\partial \psi^{2}} \cdot \frac{d \psi}{d t}+\frac{\partial U}{\partial B} \cdot \frac{\partial B}{\partial \psi} \cdot \frac{d^{2} \psi}{d t^{2}}+\frac{\partial^{2} U}{\partial t^{2}}
\end{aligned}\right.
$$

Observing that such a field holds for a kind of Levitron-type top, we may consider the external torque $L_{\theta}, L_{\varphi}, L_{\psi}$ as torque of interaction with gravitational and/or electromagnetic fields. In elliptic coordinates, the complex angles $\theta, \varphi, \psi$ are orthogonal and by analogy with the Laplace operator in elliptic coordinates, for a generalized field $U=U[\theta(t), \varphi(t), \psi(t), B[\theta, \varphi, \psi], t]$, the equation (62) becomes:

$$
\left\{\begin{array}{l}
\left(I_{\theta}+\frac{\partial B_{g}}{\partial \theta}\right) \cdot \frac{d^{2} U_{\theta}}{d t^{2}}=\frac{d^{2} U_{\theta}}{d \theta^{2}} \cdot(\wp(\varphi)-\wp(\psi)) ; \\
\left(I_{\varphi}+\frac{\partial B_{g}}{\partial \varphi}\right) \cdot \frac{d^{2} U_{\varphi}}{d t^{2}}=\frac{d^{2} U_{\varphi}}{d \varphi^{2}} \cdot(\wp(\theta)-\wp(\varphi)) ; \\
\left(I_{\psi}+\frac{\partial B_{g}}{\partial \psi}\right) \cdot \frac{d^{2} U_{\psi}}{d t^{2}}=\frac{d^{2} U_{\psi}}{d \psi^{2}} \cdot(\wp(\theta)-\wp(\varphi)) ;
\end{array}\right.
$$

where the terms: 
are the Lame coefficients, $\wp($.$) is the Weierstrass gamma function and the terms \frac{\partial(.)}{\partial .}$ are the sensitivities (of first order) of the system against the magnetic field $B_{g}$. Evidently, the interaction between a body like a Levitron-type top and the gravitational field is realized through the mean value over a precession period of the bending and restoring pulses.

In relation (65), we may consider the terms $I_{[\cdot]}$ and $\frac{\partial B}{\partial \text {. }}$ as sensitivities:

$$
\left\{\begin{array}{l}
I_{\theta} \Rightarrow S_{G \theta} ; \frac{\partial B_{g}}{\partial \theta} \Rightarrow S_{B \theta} \\
I_{\varphi} \Rightarrow S_{G \varphi} ; \frac{\partial B_{g}}{\partial \theta} \Rightarrow S_{B \varphi} \\
I_{\psi} \Rightarrow S_{G \psi} ; \frac{\partial B_{g}}{\partial \theta} \Rightarrow S_{B \psi}
\end{array}\right.
$$

From the definition of the field $U$ it results that the elliptic coordinates are the same for the gravitational and magnetic fields. Then, using (64) and (66), we can write:

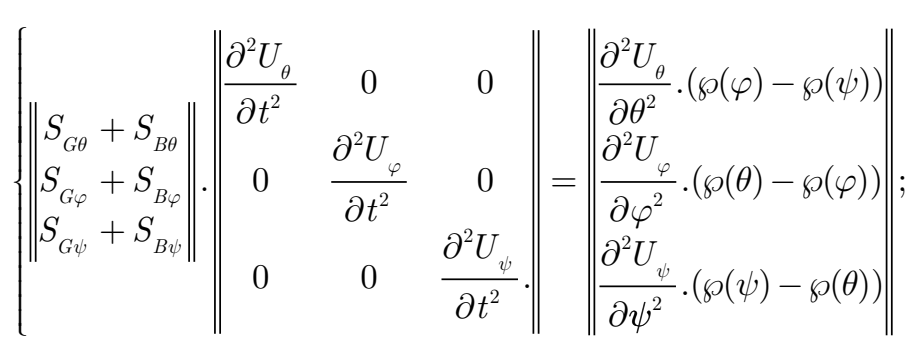

where:

$\Delta_{e}[U]=\frac{\partial^{2} U}{\partial \theta^{2}} \cdot\left(\wp(\varphi)-\wp(\psi)+\frac{\partial^{2} U}{\partial \varphi^{2}} \cdot\left(\wp(\theta)-\wp(\varphi)+\frac{\partial^{2} U}{\partial \psi^{2}} \cdot(\wp(\psi)-\wp(\theta) ;\right.\right.$

is the Laplace operator in elliptic coordinates. As the relation (65) is equivalent to the relation (68), relation (69) becomes:

$\Delta_{e}[U]=\left(S_{G}+S_{B}\right) \cdot \frac{\partial^{2} U}{\partial t^{2}}$

which formally is a waves equation in elliptic coordinates. One remark that this wave includes the gravitational/inertial and electromagnetic fields.

It can be also remarked that like for a wave equation, the coefficients: 


$$
a^{2}=S_{G}+S_{B}
$$

depends formally on the sensitivities of the considered system and may include all its properties. It can also be observed that for $m>1$, Jacobi elliptic functions are also analytic and have wave properties.

\section{CONCLUSION}

Cartesian coordinates system holds well for short distances and is based on polynomials and complex exponential functions. It is very successful in electricity, telecommunications, electronics, photonics, and even mechanics. Elliptic coordinates system holds for short, long or even very long distances and is based on elliptic functions that are a natural extension in 3D of complex exponential functions. The independent 3D variables of elliptic coordinates are homogeneous and normalized complex angles. The elliptic coordinates system uses Weierstrass and Jacobi elliptic functions to represents 1 to 4 dimensions of space and ultra-elliptic functions (Eilbeck and all, 2015) to cover 5D and more dimensions. In 4D, there exists 3 independent variables $\mathrm{u}, \mathrm{v}, \mathrm{w}$ that may 'draw' 3D volumes. Ultraelliptic functions may be represented as vector and/or tensor extensions of elliptic functions and physically, may represent other physical phenomena that cannot be 'viewed' in the 3D space that we consider now. Elliptic functions had been used in aerodynamics and hydrodynamics and now may be used in mechanics and even to represent waves and gravitational fields. One may observe that any coordinate system adds a given geometric structure to the 'space representation'. For elliptic coordinates, this geometric structure is represented by functions. As transducers are also represented by functions, one may accept that any dimension of our physical space is 'viewed' through transducers. It can be also observed that mathematically, all the elliptic functions may be built starting only from a single number, the elliptic modulus $m$. In fact, the matter that is a 'field source', may be considered as a transducer between its properties like mass, electric charge, and so on and gravitational and/or electromagnetic fields because it interacts with these fields. For humans, mathematic is a good transducer between the physical environment and our mind. We remark that the universe is 'ephemeral' but we need some physical laws that are not 'ephemeral' to be able to progress and to send our knowledge to our future generation. Actual experience shows that this is possible and probably is due to the 'granularity' of the Universe. Observing that is practically impossible to know all the parameters of a given system, statistical models may predict the 'probability of a given interaction'. Meteorological science is a good example but we may also observe that the well-known Ohm and Kirchhoff laws are also 'statistics' because it holds only for electrical currents bigger than picoamperes.

Matter, as we know it now, is a given structure of an assembly of elementary particles. It is constituted by atoms that had a given analogy with a solar system. We observe that the mathematical model for Levitron-type gyroscope toy may be used for this toy but also for some elementary atomic particle and for some celestial body. This suggests that the structure consisting of several particles that model the matter retains some of the self-similarity properties of a single particle. In Universe, interactions take place through transducers that 'connect' two and/or many items on a given (nearly similar) parameter or domain. This leads to an exchange of energy (or mass) that corresponds mathematically to 'correlation'. As interactions imply forces and these forces are generated by fields, one may consider that interactions take place between fields. Any interaction implies some conditions in which it may take place. Only if all these conditions are reunited at a given point of space and time, this 'interaction' can take place. Then, at each moment of time and point of space, a given (possible) interaction depends on the 'probability that all the necessary conditions are reunited'. This may explain the 'diversity of interaction in space' and also the 'locally diversity of space itself' but may also lead to the possibility to realize now an interaction at a given point in space-time by adding locally the necessary energy/mass to create the missing conditions and/or to stop this interaction by 
destroying some of the existing conditions. As interactions take place between fields and in some manner, fields may propagate as waves, may we suppose that an interaction takes place between waves? May all of that be put in connection with (Photonics 2019. Ultrashort-Pulse Laser Could Solve Dilemma of Nuclear Waste Disposal) because an ultrashort-pulse laser has a large frequency spectrum bandwidth and probably, some of these frequencies may interact with radioactive atoms and change their decay ratio?

Based on these ideas, the paper presents some theoretical tools that show the existing connection between transducers and elliptic functions. This may lead to a better understanding of 'inertia' and gravitational waves and, maybe, will allow to the possibility to realize transducers for frequencies greater than $3 \mathrm{EHz}$ and also a 'tractor ray. I hope that some of the (theoretical) tools presented here will be useful to develop these new technologies for ' $\mathrm{X}$ and Gamma rays' frequencies.

\section{ACKNOWLEDGMENT}

The author wishes to thanks to all the IGI Global team for their help and encouragement in writing this paper. 


\section{REFERENCES}

Ciulin, D. (2013a, January-March). Contribution to a future inertial motor and more. International Journal of Strategic Information Technology and Applications, 4(1), 63-97. doi:10.4018/jsita.2013010105 doi:10.4018/ jsita.2013010105

Ciulin, D. (2013b, July-September). About space-time and more. International Journal of Strategic Information Technology and Applications, 4(3), 1-41. doi:10.4018/ijsita.2013070101 doi:10.4018/ijsita.2013070101

Eilbeck, J. C., Enolskii, V. Z., \& Leykin, D. V. (2015). On the Kleinian construction of Abelian functions of canonical algebraic curves. Retrieved from https://www.researchgate.net/publication/2396696_On_The_ Kleinian_Construction_Of_Abelian_Functions_Of_Canonical_Algebraic_Curves

Lindsay, R. B. (1965). Physical mechanics. Princeton, NJ: Van Nostrand Reinhold.

Marcikic, I., de Riedmatten, H., Tittel, W., Zbinden, H., \& Gisin, N. (2013, January 30). Long-distance teleportation of qubits at telecommunication wavelengths. Nature, 421(6922), 509-513. doi:10.1038/nature01376 PubMed doi:10.1038/nature01376 PMID:12556886

Shannon, C. E. (1949, January). Communication in the presence of noise. Proceedings of the IRE, 37(1), 10-21. doi:10.1109/JRPROC.1949.232969 doi:10.1109/JRPROC.1949.232969

Photonics. (2014, September). Photon state teleported 25 km. Retrieved from https://www.photonics.com/ Article.aspx?AID=56699

Photonics. (2017, September). Controlling light with light. Retrieved from https://www.photonics.com/Article. aspx?AID $=62569$

Photonics. (2019). Ultrashort-Pulse Laser Could Solve Dilemma of Nuclear Waste Disposal. Academic Press.

Graitoelectromagnetism. (2015). In Wikipedia. Retrieved from https://en.wikipedia.org/wiki/ Gravitoelectromagnetism

Lie Detection. (2015). In Wikipedia. Retrieved from https://en.wikipedia.org/wiki/Lie_detection

Polaron. (2015). In Wikipedia. Retrieved from https://en.wikipedia.org/wiki/Polaron

Diophantine Equation. (2016). In Wikipedia. Retrieved from https://en.wikipedia.org/wiki/Diophantine_equation Neil Harbisson. (2016). In Wikipedia. Retrieved from https://en.wikipedia.org/wiki/Neil_Harbisson

Noether's Theorem. (2016). In Wikpedia. Retrieved from https://en.wikipedia.org/wiki/Noether\%27s_theorem Gravitational Wave. (2017). In Wikipedia. Retrieved from https://en.wikipedia.org/wiki/Gravitational_wave 